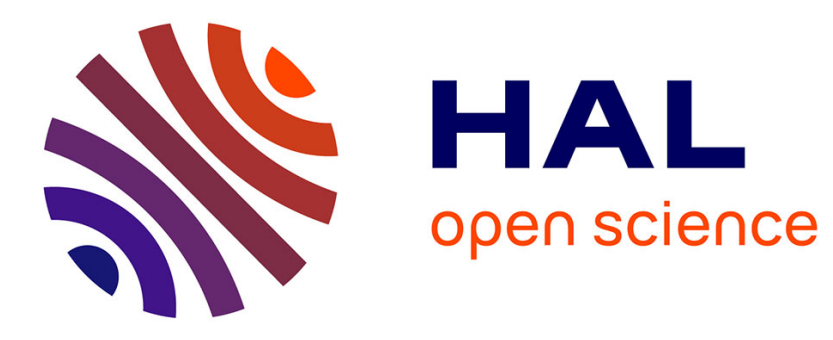

\title{
Elliptic Functions and Transcendence
}

Michel Waldschmidt

\section{To cite this version:}

Michel Waldschmidt. Elliptic Functions and Transcendence. Surveys in Number Theory, Springer Verlag, pp.143-188, 2008, Developments in Mathematics 17. hal-00407231

\section{HAL Id: hal-00407231 https://hal.science/hal-00407231}

Submitted on 24 Jul 2009

HAL is a multi-disciplinary open access archive for the deposit and dissemination of scientific research documents, whether they are published or not. The documents may come from teaching and research institutions in France or abroad, or from public or private research centers.
L'archive ouverte pluridisciplinaire HAL, est destinée au dépôt et à la diffusion de documents scientifiques de niveau recherche, publiés ou non, émanant des établissements d'enseignement et de recherche français ou étrangers, des laboratoires publics ou privés. 


\title{
Elliptic Functions and Transcendence
}

\author{
Michel Waldschmidt \\ Université P. et M. Curie (Paris VI), Institut de Mathématiques de Jussieu, \\ UMR 7586 CNRS, Problèmes Diophantiens, Case 247, 175, rue du Chevaleret F-75013 \\ Paris, France \\ miw@math.jussieu.fr; http://www.math.jussieu.fr/ miw/
}

Summary. Transcendental numbers form a fascinating subject: so little is known about the nature of analytic constants that more research is needed in this area. Even when one is interested only in numbers like $\pi$ and $e^{\pi}$ that are related to the classical exponential function, it turns out that elliptic functions are required (so far, this should not last forever!) to prove transcendence results and get a better understanding of the situation.

First we briefly recall some of the basic transcendence results related to the exponential function (Section 1). Next, in Section 2, we survey the main properties of elliptic functions that are involved in transcendence theory.

We survey transcendence theory of values of elliptic functions in Section 3, linear independence in Section 4, and algebraic independence in Section 5. This splitting is somewhat artificial but convenient. Moreover, we restrict ourselves to elliptic functions, even when many results are only special cases of statements valid for abelian functions. A number of related topics are not considered here (e.g., heights, $p$-adic theory, theta functions, Diophantine geometry on elliptic curves).

Key words: Transcendental numbers, elliptic functions, elliptic curves, elliptic integrals, algebraic independence, transcendence measures, measures of algebraic independence, Diophantine approximation.

\section{Mathematics Subject Classifications: 01-02 11G05 11J89}

Mathematical history lecture given on February 21, 2005, at the Mathematics Department of the University of Florida for the Special Year in Number Theory and Combinatorics 2004-05, supported by the France-Florida Research Institute (FFRI). The author is thankful to Krishna Alladi for his invitation to deliver the lecture and for his suggestion of coming up with a written version. He is also grateful to Nikos Tzanakis for his proposal to deliver a course for the Pichorides Distinguished Lectureship funded by FORTH (Foundation of Research and Technology Hellas) during the summer 2005 at the Department of Mathematics, University of Crete, where this survey was written. Last but not least, many thanks to F. Amoroso, D. Bertrand, N. Brisebarre, P. Bundschuh, E. Gaudron, N. Hirata-Kohno, C. Levesque, D. W. Masser, F. \& R. Pellarin, P. Philippon, I. Wakabayashi, and all colleagues who contributed their helpful comments on preliminary versions of this survey. Université P. et M. Curie (Paris VI). http://www.math.jussieu.fr/ miw/ 


\section{Exponential Function and Transcendence}

We start with a very brief list of some of the main transcendence results concerning numbers related to the exponential function. References are, for instance, $[13,83$, 89, 120, 192, 207, 211, 233].

Next, we point out some properties of the exponential function, the elliptic analogue of which we shall consider later (Section 2.1).

\subsection{Short Survey on the Transcendence of Numbers Related to the Exponential Function}

\section{Hermite, Lindemann, and Weierstrass}

The first transcendence result for a number related to the exponential function is Hermite's theorem on the transcendence of $e$.

Theorem 1 (Hermite, 1873). The number e is transcendental.

This means that for any nonzero polynomial $P \in \mathbb{Z}[X]$, the number $P(e)$ is not zero. We denote by $\overline{\mathbb{Q}}$ the set of algebraic numbers. Hence Hermite's theorem can be written $e \notin \overline{\mathbb{Q}}$. A complex number is called transcendental if it is transcendental over $\mathbb{Q}$, or over $\overline{\mathbb{Q}}$, which is the same. Also we shall say that complex numbers $\theta_{1}, \ldots, \theta_{n}$ are algebraically independent if they are algebraically independent over $\mathbb{Q}$, which is the same as over $\overline{\mathbb{Q}}$ : for any nonzero polynomial $P$ in $n$ variables (and coefficients in $\mathbb{Z}, \mathbb{Q}$, or $\overline{\mathbb{Q}})$, the number $P\left(\theta_{1}, \ldots, \theta_{n}\right)$ is not zero.

The second result in chronological order is Lindemann's theorem on the transcendence of $\pi$.

Theorem 2 (Lindemann, 1882). The number $\pi$ is transcendental.

The next result contains the transcendence of both numbers $e$ and $\pi$ :

Theorem 3 (Hermite-Lindemann, 1882). For $\alpha \in \overline{\mathbb{Q}}^{\times}$, any nonzero logarithm $\log \alpha$ of $\alpha$ is transcendental.

We denote by $\mathcal{L}$ the $\mathbb{Q}$-vector space of logarithms of algebraic numbers:

$$
\mathcal{L}=\left\{\log \alpha ; \alpha \in \overline{\mathbb{Q}}^{\times}\right\}=\left\{\ell \in \mathbb{C} ; e^{\ell} \in \overline{\mathbb{Q}}^{\times}\right\}=\exp ^{-1}\left(\overline{\mathbb{Q}}^{\times}\right) .
$$

Hence Theorem 3 means that $\mathcal{L} \cap \overline{\mathbb{Q}}=\{0\}$. An alternative form is the following:

Theorem 4 (Hermite-Lindemann, 1882). For any $\beta \in \overline{\mathbb{Q}}^{\times}$, the number $e^{\beta}$ is transcendental.

The first result of algebraic independence for the values of the exponential function goes back to the end of the nineteenth century. 
Theorem 5 (Lindemann-Weierstrass, 1885). Let $\beta_{1}, \ldots, \beta_{n}$ be $\mathbb{Q}$-linearly independent algebraic numbers. Then the numbers $e^{\beta_{1}}, \ldots, e^{\beta_{n}}$ are algebraically independent.

Again, there is an alternative form of Theorem 5: it amounts to a statement of linear independence.

Theorem 6 (Lindemann-Weierstrass, 1885). Let $\gamma_{1}, \ldots, \gamma_{m}$ be distinct algebraic numbers. Then the numbers $e^{\gamma_{1}}, \ldots, e^{\gamma_{m}}$ are linearly independent over $\overline{\mathbb{Q}}$.

It is not difficult to check that Theorem 6 is equivalent to Theorem 5 with the conclusion that $e^{\beta_{1}}, \ldots, e^{\beta_{n}}$ are algebraically independent over $\overline{\mathbb{Q}}$; since it is equivalent to saying that $e^{\beta_{1}}, \ldots, e^{\beta_{n}}$ are algebraically independent over $\mathbb{Q}$, one does not lose anything if one changes the conclusion of Theorem 6 by stating that the numbers $e^{\gamma_{1}}, \ldots, e^{\gamma_{m}}$ are linearly independent over $\mathbb{Q}$.

\section{Hilbert's Seventh Problem, Gel'fond and Schneider}

The solution of Hilbert's seventh problem on the transcendence of $\alpha^{\beta}$ was obtained by Gel'fond and Schneider in 1934 (see [89, 207]).

Theorem 7 (Gel'fond-Schneider, 1934). For $\alpha$ and $\beta$ algebraic numbers with $\alpha \neq 0$ and $\beta \notin \mathbb{Q}$ and for any choice of $\log \alpha \neq 0$, the number $\alpha^{\beta}=\exp (\beta \log \alpha)$ is transcendental.

This means that the two algebraically independent functions $e^{z}$ and $e^{\beta z}$ cannot take algebraic values at the points $\log \alpha$ (A.O. Gel'fond) and also that the two algebraically independent functions $z$ and $\alpha^{z}=e^{z \log \alpha}$ cannot take algebraic values at the points $m+n \beta$ with $(m, n) \in \mathbb{Z}^{2}$ (Th. Schneider).

Examples (quoted by D. Hilbert in 1900) of numbers whose transcendence follows from Theorem 7 are $2^{\sqrt{2}}$ and $e^{\pi}$ (recall that $e^{i \pi}=-1$ ). The transcendence of $e^{\pi}$ had already been proved in 1929 by A.O. Gel'fond.

Here is an equivalent statement to Theorem 7:

Theorem 8 (Gel'fond-Schneider, 1934). Let $\log \alpha_{1}, \log \alpha_{2}$ be two nonzero logarithms of algebraic numbers. Assume that the quotient $\left(\log \alpha_{1}\right) /\left(\log \alpha_{2}\right)$ is irrational. Then this quotient is transcendental.

\section{Linear Independence of Logarithms of Algebraic Numbers}

The generalization of Theorem 8 to more than two logarithms, conjectured by A.O. Gel'fond [89], was proved by A. Baker in 1966. His results include not only Theorem 8 but also Theorem 3 .

Theorem 9 (Baker, 1966). Let $\log \alpha_{1}, \ldots, \log \alpha_{n}$ be $\mathbb{Q}$-linearly independent logarithms of algebraic numbers. Then the numbers $1, \log \alpha_{1}, \ldots, \log \alpha_{n}$ are linearly independent over the field $\overline{\mathbb{Q}}$. 


\section{The Six Exponentials Theorem and the Four Exponentials Conjecture}

The next result, which does not follow from any of the previously mentioned results, was proved independently in the 1940s by C.L. Siegel (unpublished) and in the 1960s by S. Lang and K. Ramachandra (see [120, 191, 238]; see also Problem 1 in [207] for the four exponentials conjecture). As suggested by K. Ramachandra (see [192] Section 3.1, Theorem 2), Theorem 10 also follows from Schneider's criterion proved in 1949 [206].

Theorem 10 (Six Exponentials Theorem). Let $x_{1}, \ldots, x_{d}$ be $\mathbb{Q}$-linearly independent complex numbers and let $y_{1}, \ldots, y_{\ell}$ be $\mathbb{Q}$-linearly independent complex numbers. Assume $\ell d>\ell+d$. Then at least one of the $\ell d$ numbers

$$
e^{x_{i} y_{j}} \quad(1 \leq i \leq d, 1 \leq j \leq \ell)
$$

is transcendental.

Notice that the condition $\ell d>\ell+d$ can be written $(\ell \geq 2$ and $d \geq 3)$ or $(\ell \geq 3$ and $d \geq 2$ ); it suffices to consider the case $\ell d=6$ (hence the name of the result). Therefore, Theorem 10 can be stated in an equivalent form:

Theorem 11 (Six Exponentials Theorem-logarithmic form). Let

$$
M=\left(\begin{array}{l}
\log \alpha_{1} \log \alpha_{2} \log \alpha_{3} \\
\log \beta_{1} \log \beta_{2} \log \beta_{3}
\end{array}\right)
$$

be a 2-by-3 matrix whose entries are logarithms of algebraic numbers. Assume that the three columns are linearly independent over $\mathbb{Q}$ and the two rows are also linearly independent over $\mathbb{Q}$. Then the matrix $M$ has rank 2 .

It is expected that the condition $d \ell>d+\ell$ in Theorem 10 is too restrictive and that the same conclusion holds in the case $d=\ell=2$. We state this conjecture in the logarithmic form:

Conjecture 12 (Four exponentials conjecture - logarithmic form). Let

$$
M=\left(\begin{array}{l}
\log \alpha_{1} \log \alpha_{2} \\
\log \beta_{1} \log \beta_{2}
\end{array}\right)
$$

be a 2-by-2 matrix whose entries are logarithms of algebraic numbers. Assume that the two columns are linearly independent over $\mathbb{Q}$ and that the two rows are also linearly independent over $\mathbb{Q}$. Then the matrix $M$ has rank 2 .

\section{Algebraic Independence}

In 1948 and 1949, A.O. Gel'fond extended his solution of Hilbert's seventh problem to a result of algebraic independence [89]. One of his theorems is that the two 
numbers $2^{\sqrt[3]{2}}$ and $2^{\sqrt[3]{4}}$ are algebraically independent. His general statements can be seen as extensions of Theorem 10 into a result of algebraic independence (in spite of the fact that Theorem 10 was stated and proved only several years later). In his original work, Gel'fond needed a stronger assumption, namely a measure of linear independence of the $x_{i}$ 's as well as of the $y_{j}$ 's. This assumption was removed in the early 1970s by R. Tijdeman [217] (further references, especially to papers by A.A. Smelev and W.D. Brownawell, are given in [230]; see also [35, 235, 236, 242]).

Theorem 13. Let $x_{1}, \ldots, x_{d}$ be $\mathbb{Q}$-linearly independent complex numbers and let $y_{1}, \ldots, y_{\ell}$ be $\mathbb{Q}$-linearly independent complex numbers.

1. If $d \ell \geq 2(d+\ell)$, then at least two of the $d \ell$ numbers

$$
e^{x_{i} y_{j}} \quad(1 \leq i \leq d, 1 \leq j \leq \ell)
$$

are algebraically independent.

2. If $d \ell \geq d+2 \ell$, then at least two of the $d \ell+d$ numbers

$$
x_{i}, e^{x_{i} y_{j}} \quad(1 \leq i \leq d, 1 \leq j \leq \ell)
$$

are algebraically independent.

3. If $d \ell>d+\ell$, then at least two of the $d \ell+d+\ell$ numbers

$$
x_{i}, y_{j}, e^{x_{i} y_{j}} \quad(1 \leq i \leq d, 1 \leq j \leq \ell)
$$

are algebraically independent.

4. If $d=\ell=2$ and if the two numbers $e^{x_{1} y_{1}}$ and $e^{x_{1} y_{2}}$ are algebraic, then at least two of the six numbers

$$
x_{1}, x_{2}, y_{1}, y_{2}, e^{x_{2} y_{1}}, e^{x_{2} y_{2}}
$$

are algebraically independent.

From the last part of Theorem 13, taking $x_{1}=y_{1}=i \pi$ and $x_{2}=y_{2}=1$, one deduces that at least one of the two following statements is true:

(i) The number $e^{\pi^{2}}$ is transcendental.

(ii) The two numbers $e$ and $\pi$ are algebraically independent.

One expects that both statements are true.

If it were possible to prove that under the assumptions of Theorem 13, at least two of the eight numbers

$$
x_{1}, x_{2}, y_{1}, y_{2}, e^{x_{1} y_{1}}, e^{x_{1} y_{2}}, e^{x_{2} y_{1}}, e^{x_{2} y_{2}}
$$

are algebraically independent, one would deduce the algebraic independence of the two numbers $\pi$ and $e^{\pi}$ (take $x_{1}=1, x_{2}=i, y_{1}=\pi, y_{2}=i \pi$; see Corollary 48 below).

For results concerning large transcendence degree, see Section 5.3 below. 


\subsection{The Exponential Function}

The exponential function

$$
\begin{aligned}
\exp : \mathbb{C} & \rightarrow \mathbb{C}^{\times}, \\
z & \mapsto e^{z},
\end{aligned}
$$

satisfies both a differential equation and an addition formula:

$$
\frac{d}{d z} e^{z}=e^{z}, \quad e^{z_{1}+z_{2}}=e^{z_{1}} e^{z_{2}} .
$$

It is a homomorphism of the additive group $\mathbb{C}$ of complex numbers onto the multiplicative group $\mathbb{C}^{\times}$of nonzero complex numbers, with kernel

$$
\text { ker } \exp =2 \pi i \mathbb{Z}
$$

Hence it yields an isomorphism between the quotient additive group $\mathbb{C} / 2 \pi i \mathbb{Z}$ and the multiplicative group $\mathbb{C}^{\times}$.

The group $\mathbb{C}^{\times}$is the group of complex points of the multiplicative group $\mathbb{G}_{m}$; $z \mapsto e^{z}$ is the exponential function of the multiplicative group $\mathbb{G}_{m}$. We shall replace this algebraic group by an elliptic curve. We could replace it also by other commutative algebraic groups. As a first example, the exponential function of the additive group $\mathbb{G}_{a}$ is

$$
\begin{aligned}
& \mathbb{C} \rightarrow \mathbb{C}, \\
& z \mapsto z .
\end{aligned}
$$

More general examples are commutative linear algebraic groups; over an algebraically closed field, these are nothing else than products of several copies of the additive and multiplicative group. Further examples of algebraic groups are abelian varieties. In full generality, algebraic groups are extensions of abelian varieties by commutative linear algebraic groups. See, for instance, [120, 158, 233].

\section{Elliptic Curves and Elliptic Functions}

Among many references for this section are the books by S. Lang [127], K. Chandrasekharan [43], and J. Silverman [212, 213]. See also the book by M. Hindry and J. Silverman [99].

\subsection{Basic Concepts}

An elliptic curve may be defined as

- $y^{2}=C(x)$ for a square-free cubic polynomial $C(x)$,

- a connected compact Lie group of dimension 1 ,

- a complex torus $\mathbb{C} / \Omega$, where $\Omega$ is a lattice in $\mathbb{C}$,

- a Riemann surface of genus 1 , 
- a nonsingular cubic in $\mathbb{P}_{2}(\mathbb{C})$ (together with a point at infinity),

- an algebraic group of dimension 1, with underlying projective algebraic variety.

We shall use the Weierstrass form

$$
E=\left\{(t: x: y) ; y^{2} t=4 x^{3}-g_{2} x t^{2}-g_{3} t^{3}\right\} \subset \mathbb{P}_{2} .
$$

Here $g_{2}$ and $g_{3}$ are complex numbers, with the only assumption $g_{2}^{3} \neq 27 g_{3}^{2}$, which means that the discriminant of the polynomial $4 X^{3}-g_{2} X-g_{3}$ does not vanish.

An analytic parametrization of the complex points $E(\mathbb{C})$ of $E$ is given by means of the Weierstrass elliptic function $\wp$, which satisfies the differential equation

$$
\wp^{\prime 2}=4 \wp^{3}-g_{2} \wp-g_{3} .
$$

It has a double pole at the origin with principal part $1 / z^{2}$ and also satisfies an addition formula

$$
\wp\left(z_{1}+z_{2}\right)=-\wp\left(z_{1}\right)-\wp\left(z_{2}\right)+\frac{1}{4} \cdot\left(\frac{\wp^{\prime}\left(z_{1}\right)-\wp^{\prime}\left(z_{2}\right)}{\wp\left(z_{1}\right)-\wp\left(z_{2}\right)}\right)^{2} .
$$

The exponential map of the Lie group $E(\mathbb{C})$ is

$$
\begin{aligned}
\exp _{E}: \mathbb{C} & \rightarrow E(\mathbb{C}), \\
z & \mapsto\left(1: \wp(z): \wp^{\prime}(z)\right) .
\end{aligned}
$$

The kernel of this map is a lattice in $\mathbb{C}$ (that is, a discrete rank-2 subgroup),

$$
\Omega=\operatorname{ker} \exp _{E}=\{\omega \in \mathbb{C} ; \wp(z+\omega)=\wp(z)\}=\mathbb{Z} \omega_{1}+\mathbb{Z} \omega_{2} .
$$

Hence $\exp _{E}$ induces an isomorphism between the quotient additive group $\mathbb{C} / \Omega$ and $E(\mathbb{C})$ with the law given by (2). The elements of $\Omega$ are the periods of $\wp$. A pair $\left(\omega_{1}, \omega_{2}\right)$ of fundamental periods is given by (cf. [244] Section 20.32, Example 1)

$$
\omega_{i}=2 \int_{e_{i}}^{\infty} \frac{d x}{\sqrt{4 x^{3}-g_{2} x-g_{3}}} \quad(i=1,2),
$$

where

$$
4 x^{3}-g_{2} x-g_{3}=4\left(x-e_{1}\right)\left(x-e_{2}\right)\left(x-e_{3}\right) .
$$

Indeed, since $\wp^{\prime}$ is periodic and odd, it vanishes at $\omega_{1} / 2, \omega_{2} / 2$ and $\left(\omega_{1}+\omega_{2}\right) / 2$; hence the values of $\wp$ at these points are the three distinct complex numbers $e_{1}, e_{2}$, and $e_{3}$ (recall that the discriminant of $4 x^{3}-g_{2} x-g_{3}$ is not 0 ).

Conversely, given a lattice $\Omega$, there is a unique Weierstrass elliptic function $\wp_{\Omega}$ whose period lattice is $\Omega$ (see Section 2.5). We denote its invariants in the differential equation $(1)$ by $g_{2}(\Omega)$ and $g_{3}(\Omega)$.

We shall be interested mainly (but not only) in elliptic curves that are defined over the field of algebraic numbers: they have a Weierstrass equation with algebraic 
$g_{2}$ and $g_{3}$. However, we shall also use the Weierstrass elliptic function associated with the lattice $\lambda \Omega$, where $\lambda \in \mathbb{C}^{\times}$may be transcendental; the relations are

$$
\wp_{\lambda \Omega}(\lambda z)=\lambda^{-2} \wp_{\Omega}(z), \quad g_{2}(\lambda \Omega)=\lambda^{-4} g_{2}(\Omega), \quad g_{3}(\lambda \Omega)=\lambda^{-6} g_{3}(\Omega) .
$$

The lattice $\Omega=\mathbb{Z}+\mathbb{Z} \tau$, where $\tau$ is a complex number with positive imaginary part, satisfies

$$
g_{2}(\mathbb{Z}+\mathbb{Z} \tau)=60 G_{2}(\tau) \quad \text { and } \quad g_{3}(\mathbb{Z}+\mathbb{Z} \tau)=140 G_{3}(\tau),
$$

where $G_{k}(\tau)$ (with $k \geq 2$ ) are the Eisenstein series (see, for instance, [48] Section 3.2, [208] Section 7.2.3, [116] Section 3.2 or [212] Section 6.3 - the normalization in [254] p. 240 is different):

$$
G_{k}(\tau)=\sum_{(m, n) \in \mathbb{Z}^{2} \backslash\{(0,0)\}}(m+n \tau)^{-2 k}
$$

\subsection{Morphisms between Elliptic Curves. The Modular Invariant}

If $\Omega$ and $\Omega^{\prime}$ are two lattices in $\mathbb{C}$ and if $f: \mathbb{C} / \Omega \rightarrow \mathbb{C} / \Omega^{\prime}$ is an analytic homomorphism, then the map $\mathbb{C} \rightarrow \mathbb{C} / \Omega \rightarrow \mathbb{C} / \Omega^{\prime}$ factors through a homothecy $\mathbb{C} \rightarrow \mathbb{C}$ given by some $\lambda \in \mathbb{C}$ such that $\lambda \Omega \subset \Omega^{\prime}$ :

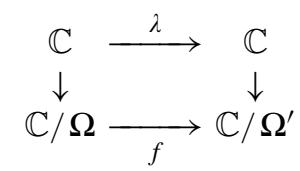

If $f \neq 0$, then $\lambda \in \mathbb{C}^{\times}$and $f$ is surjective.

Conversely, if there exists $\lambda \in \mathbb{C}$ such that $\lambda \Omega \subset \Omega^{\prime}$, then $f_{\lambda}(x+\Omega)=\lambda x+\Omega^{\prime}$ defines an analytic surjective homomorphism $f_{\lambda}: \mathbb{C} / \Omega \rightarrow \mathbb{C} / \Omega^{\prime}$. In this case $\lambda \Omega$ is a subgroup of finite index in $\Omega^{\prime}$; hence the kernel of $f_{\lambda}$ is finite and there exists $\mu \in \mathbb{C}^{\times}$with $\mu \Omega^{\prime} \subset \Omega$ : the two elliptic curves $\mathbb{C} / \Omega$ and $\mathbb{C} / \Omega^{\prime}$ are isogenous.

If $\Omega$ and $\Omega^{*}$ are two lattices, $\wp$ and $\wp^{*}$ the associated Weierstrass elliptic functions, and $g_{2}, g_{3}$ the invariants of $\wp$, the following statements are equivalent:

(i) There is a $2 \times 2$ matrix with rational coefficients that maps a basis of $\Omega$ to a basis of $\Omega^{*}$.

(ii) There exists $\lambda \in \mathbb{Q}^{\times}$such that $\lambda \Omega \subset \Omega^{*}$.

(iii) There exists $\lambda \in \mathbb{Z} \backslash\{0\}$ such that $\lambda \Omega \subset \Omega^{*}$.

(iv) The two functions $\wp$ and $\wp^{*}$ are algebraically dependent over the field $\mathbb{Q}\left(g_{2}, g_{3}\right)$.

(v) The two functions $\wp$ and $\wp^{*}$ are algebraically dependent over $\mathbb{C}$.

The map $f_{\lambda}$ is an isomorphism if and only if $\lambda \Omega=\Omega^{\prime}$.

The number

$$
j=\frac{1728 g_{2}^{3}}{g_{2}^{3}-27 g_{3}^{2}}
$$


is the modular invariant of the elliptic curve $E$. Two elliptic curves over $\mathbb{C}$ are isomorphic if and only if they have the same modular invariant.

$$
\begin{aligned}
& \text { Set } \tau=\omega_{2} / \omega_{1}, q=e^{2 \pi i \tau} \text { and } J\left(e^{2 \pi i \tau}\right)=j(\tau) \text {. Then } \\
& \qquad \begin{aligned}
J(q) & =q^{-1}\left(1+240 \sum_{m=1}^{\infty} m^{3} \frac{q^{m}}{1-q^{m}}\right)^{3} \prod_{n=1}^{\infty}\left(1-q^{n}\right)^{-24} \\
& =\frac{1}{q}+744+196884 q+21493760 q^{2}+\cdots .
\end{aligned}
\end{aligned}
$$

See [142] Section 4.12 or [208] Sections 7.3.3 and 7.4.

\subsection{Endomorphisms of an Elliptic Curve; Complex Multiplication}

Let $\Omega$ be a lattice in $\mathbb{C}$. The set of analytic endomorphisms of $\mathbb{C} / \Omega$ is the subring

$$
\operatorname{End}(\mathbb{C} / \Omega)=\left\{f_{\lambda} ; \lambda \in \mathbb{C} \text { with } \lambda \Omega \subset \Omega\right\}
$$

of $\mathbb{C}$. We also call it the ring of endomorphisms of the associated elliptic curve, or of the corresponding Weierstrass $\wp$ function, and we identify it with the subring

$$
\{\lambda \in \mathbb{C} ; \lambda \Omega \subset \Omega\}
$$

of $\mathbb{C}$. The field of endomorphisms is the quotient field $\operatorname{End}(\mathbb{C} / \Omega) \otimes_{\mathbb{Z}} \mathbb{Q}$ of this ring.

If $\lambda \in \mathbb{C}$ satisfies $\lambda \Omega \subset \Omega$, then $\lambda$ is either a rational integer or an algebraic integer in an imaginary quadratic field. For such a $\lambda, \wp_{\Omega}(\lambda z)$ is a rational function of $\wp_{\Omega}(z)$; the degree of the numerator is $\lambda^{2}$ if $\lambda \in \mathbb{Z}$ and $N(\lambda)$ otherwise (here, $N$ is the norm of the imaginary quadratic field); the degree of the denominator is $\lambda^{2}-1$ if $\lambda \in \mathbb{Z}$ and $N(\lambda)-1$ otherwise.

Let $E$ be the elliptic curve attached to the Weierstrass $\wp$ function. The ring of endomorphisms $\operatorname{End}(E)$ of $E$ is either $\mathbb{Z}$ or an order in an imaginary quadratic field $k$. The latter case arises if and only if the quotient $\tau=\omega_{2} / \omega_{1}$ of a pair of fundamental periods is a quadratic number; in this case the field of endomorphisms of $E$ is $k=$ $\mathbb{Q}(\tau)$ and the curve $E$ has complex multiplication; this is the so-called $C M$ case. This means also that the two functions $\wp(z)$ and $\wp(\tau z)$ are algebraically dependent. In this case, the value $j(\tau)$ of the modular invariant $j$ is an algebraic integer whose degree is the class number of the quadratic field $k=\mathbb{Q}(\tau)$.

Remark 14. From Theorem 7 one deduces the transcendence of the number

$$
e^{\pi \sqrt{163}}=262537412640768743.99999999999925007259 \ldots
$$

If we set

$$
\tau=\frac{1+i \sqrt{163}}{2}, \quad q=e^{2 \pi i \tau}=-e^{-\pi \sqrt{163}},
$$

then the class number of the imaginary quadratic field $\mathbb{Q}(\tau)$ is 1 , we have $j(\tau)=$ $-(640320)^{3}$, and 


$$
\left|j(\tau)-\frac{1}{q}-744\right|<10^{-12}
$$

Also ([57] Section 2.4)

$$
\left(e^{\pi \sqrt{163}}-744\right)^{1 / 3}=640319.99999999999999999999999939031 \ldots
$$

Let $\wp$ be a Weierstrass elliptic function with field of endomorphisms $k$. Hence $k=\mathbb{Q}$ if the associated elliptic curve has no complex multiplication, while in the other case $k$ is an imaginary quadratic field, namely $k=\mathbb{Q}(\tau)$, where $\tau$ is the quotient of two linearly independent periods of $\wp$. Let $u_{1}, \ldots, u_{d}$ be nonzero complex numbers. Then the functions $\wp\left(u_{1} z\right), \ldots, \wp\left(u_{d} z\right)$ are algebraically independent (over $\mathbb{C}$ or over $\mathbb{Q}\left(g_{2}, g_{3}\right)$; this is equivalent) if and only if the numbers $u_{1}, \ldots, u_{d}$ are linearly independent over $k$. This generalizes the fact that $\wp(z)$ and $\wp(\tau z)$ are algebraically dependent if and only if the elliptic curve has complex multiplication. Much more general and deeper results of algebraic independence of functions (exponential and elliptic functions, zeta functions, ...) were proved by W.D. Brownawell and K.K. Kubota [37].

If $\wp$ is a Weierstrass elliptic function with algebraic invariants $g_{2}$ and $g_{3}$, if $E$ is the associated elliptic curve, and if $k$ denotes its field of endomorphisms, then the set

$$
\mathcal{L}_{E}=\Omega \cup\{u \in \mathbb{C} \backslash \Omega ; \wp(u) \in \overline{\mathbb{Q}}\}
$$

is a $k$-vector subspace of $\mathbb{C}$ : this is the set of elliptic logarithms of algebraic points on $E$. It plays a role with respect to $E$ similar to the role of $\mathcal{L}$ for the multiplicative group $\mathbb{G}_{m}$.

Let $k=\mathbb{Q}(\sqrt{-d})$ be an imaginary quadratic field with class number $h(-d)=h$. There are $h$ nonisomorphic elliptic curves $E_{1}, \ldots, E_{h}$ with ring of endomorphisms the ring of integers of $k$. The numbers $j\left(E_{i}\right)$ are conjugate algebraic integers of degree $h$; each of them generates the Hilbert class field $H$ of $k$ (maximal unramified abelian extension of $k$ ). The Galois group of $H / k$ is isomorphic to the ideal class group of $k$.

Since the group of roots of units of an imaginary quadratic field is $\{-1,+1\}$ except for $\mathbb{Q}(i)$ and $\mathbb{Q}(\varrho)$, where $\varrho=e^{2 \pi i / 3}$, it follows that there are exactly two elliptic curves over $\mathbb{Q}$ (up to isomorphism) having an automorphism group bigger than $\{-1,+1\}$. They correspond to Weierstrass elliptic functions $\wp$ for which there exists a complex number $\lambda \neq \pm 1$ with $\lambda^{2} \wp(\lambda z)=\wp(z)$.

The first one has $g_{3}=0$ and $j=1728$. An explicit value for a pair of fundamental periods of the elliptic curve

$$
y^{2} t=4 x^{3}-4 x t^{2}
$$

follows from computations by Legendre using Gauss's lemniscate function ([244] Section 22.8) and yields (see [4], as well as Appendix 1 of [241])

$$
\omega_{1}=\int_{1}^{\infty} \frac{d x}{\sqrt{x^{3}-x}}=\frac{1}{2} B(1 / 4,1 / 2)=\frac{\Gamma(1 / 4)^{2}}{2^{3 / 2} \pi^{1 / 2}} \quad \text { and } \quad \omega_{2}=i \omega_{1} .
$$


The lattice $\mathbb{Z}[i]$ has $g_{2}=4 \omega_{1}^{4}$. Thus

$$
\sum_{(m, n) \in \mathbb{Z}^{2} \backslash\{(0,0)\}}(m+n i)^{-4}=\frac{\Gamma(1 / 4)^{8}}{2^{6} \cdot 3 \cdot 5 \cdot \pi^{2}} .
$$

The second one has $g_{2}=0$ and $j=0$. Again from computations by Legendre ([244] Section 22.81 II) one deduces that a pair of fundamental periods of the elliptic curve

$$
y^{2} t=4 x^{3}-4 t^{3}
$$

is (see once more [4] and Appendix 1 of [241])

$$
\omega_{1}=\int_{1}^{\infty} \frac{d x}{\sqrt{x^{3}-1}}=\frac{1}{3} B(1 / 6,1 / 2)=\frac{\Gamma(1 / 3)^{3}}{2^{4 / 3} \pi} \quad \text { and } \quad \omega_{2}=\varrho \omega_{1} .
$$

The lattice $\mathbb{Z}[\varrho]$ has $g_{3}=4 \omega_{1}^{6}$. Thus

$$
\sum_{(m, n) \in \mathbb{Z}^{2} \backslash\{(0,0)\}}(m+n \varrho)^{-6}=\frac{\Gamma(1 / 3)^{18}}{2^{8} \cdot 5 \cdot 7 \cdot \pi^{6}} .
$$

These two examples involve special values of Euler's gamma function

$$
\Gamma(z)=\int_{0}^{\infty} e^{-t} t^{z} \cdot \frac{d t}{t}=e^{-\gamma z} z^{-1} \prod_{n=1}^{\infty}\left(1+\frac{z}{n}\right)^{-1} e^{z / n},
$$

where

$$
\gamma=\lim _{n \rightarrow \infty}\left(\sum_{k=1}^{n} \frac{1}{k}-\log n\right)=0.57721566490153286060651209 \ldots
$$

is Euler's constant (Section 12.1 in [244]), while Euler's beta function is

$$
B(a, b)=\frac{\Gamma(a) \Gamma(b)}{\Gamma(a+b)}=\int_{0}^{1} x^{a-1}(1-x)^{b-1} d x .
$$

More generally, the formula of Chowla and Selberg (1966) [44] (see also [9, 95, 96, $115,117,234]$ for related results) expresses periods of elliptic curves with complex multiplication as products of gamma values: if $k$ is an imaginary quadratic field and $\mathcal{O}$ an order in $k$, if $E$ is an elliptic curve with complex multiplication by $\mathcal{O}$, then the corresponding lattice $\Omega$ determines a vector space $\Omega \otimes_{\mathbb{Z}} \mathbb{Q}$ that is invariant under the action of $k$ and thus has the form $k \cdot \omega$ for some $\omega \in \mathbb{C}^{\times}$defined up to elements in $k^{\times}$. In particular, if $\mathcal{O}$ is the ring of integers $\mathbb{Z}_{k}$ of $k$, then

$$
\omega=\alpha \sqrt{\pi} \prod_{\substack{0<a<d \\(a, d)=1}} \Gamma(a / d)^{w \epsilon(a) / 4 h},
$$

where $\alpha$ is a nonzero algebraic number, $w$ is the number of roots of unity in $k, h$ is the class number of $k$, and $\epsilon$ is the Dirichlet character modulo the discriminant $d$ of $k$. 


\subsection{Standard Relations among Gamma Values}

Euler's gamma function satisfies the following relations ([244] Chapter XII): (Translation)

$$
\Gamma(z+1)=z \Gamma(z) ;
$$

(Reflection)

$$
\Gamma(z) \Gamma(1-z)=\frac{\pi}{\sin (\pi z)} ;
$$

(Multiplication) For any positive integer $n$,

$$
\prod_{k=0}^{n-1} \Gamma\left(z+\frac{k}{n}\right)=(2 \pi)^{(n-1) / 2} n^{-n z+(1 / 2)} \Gamma(n z) .
$$

D. Rohrlich conjectured that any multiplicative relation among gamma values is a consequence of these standard relations, while S. Lang was more optimistic (see [125], [128] I Chapter 2 Appendix p. 66 and [9] Chapter 24):

Conjecture 15 (D. Rohrlich). Any multiplicative relation

$$
\pi^{b / 2} \prod_{a \in \mathbb{Q}} \Gamma(a)^{m_{a}} \in \overline{\mathbb{Q}}
$$

with $b$ and $m_{a}$ in $\mathbb{Z}$ is a consequence of the standard relations.

Conjecture 16 (S. Lang). Any algebraic dependence relation with algebraic coefficients among the numbers $(2 \pi)^{-1 / 2} \Gamma(a)$ with $a \in \mathbb{Q}$ is in the ideal generated by the standard relations.

\subsection{Quasiperiods of Elliptic Curves and Elliptic Integrals of the Second Kind}

Let $\Omega=\mathbb{Z} \omega_{1}+\mathbb{Z} \omega_{2}$ be a lattice in $\mathbb{C}$. The Weierstrass canonical product attached to this lattice is the entire function $\sigma_{\Omega}$ defined by ([244] Section 20.42)

$$
\sigma_{\Omega}(z)=z \prod_{\omega \in \Omega \backslash\{0\}}\left(1-\frac{z}{\omega}\right) e^{\frac{z}{\omega}+\frac{z^{2}}{2 \omega^{2}}} .
$$

It has a simple zero at any point of $\Omega$.

Hence the Weierstrass sigma function plays, for the lattice $\Omega$, the role that is played by the function

$$
z \prod_{n \geq 1}\left(1-\frac{z}{n}\right) e^{z / n}=-e^{\gamma z} \Gamma(-z)^{-1}
$$

for the set of positive integers $\mathbb{N} \backslash\{0\}=\{1,2, \ldots\}$ (see the infinite product (7) for Euler's gamma function), and also by the function 


$$
\pi^{-1} \sin (\pi z)=z \prod_{n \in \mathbb{Z} \backslash\{0\}}\left(1-\frac{z}{n}\right) e^{z / n}
$$

for the set $\mathbb{Z}$ of rational integers ([43] Section 4.2).

The Weierstrass sigma function $\sigma$ associated with a lattice in $\mathbb{C}$ is an entire function of order 2:

$$
\limsup _{r \rightarrow \infty} \frac{1}{\log r} \cdot \log \log \sup _{|z|=r}|\sigma(z)|=2 ;
$$

the product $\sigma^{2} \wp$ is also an entire function of order 2 (this can be checked using infinite products, but it is easier to use the quasiperiodicity of $\sigma$, see formula (8) below).

The logarithmic derivative of the sigma function is the Weierstrass zeta function $\zeta=\sigma^{\prime} / \sigma$ whose Laurent expansion at the origin is ([127] Section 18.3, [208] Section 7.2.3 and [212] Section 6.3, Theorem 3.5)

$$
\zeta(z)=\frac{1}{z}-\sum_{k \geq 2} s_{k} z^{2 k-1}
$$

where for $k \in \mathbb{Z}, k \geq 2$,

$$
s_{k}=s_{k}(\Omega)=\sum_{\substack{\omega \in \Omega \\ \omega \neq 0}} \omega^{-2 k}=\omega_{1}^{-2 k} G_{k}(\tau)
$$

(recall (4); also $\left.\tau=\omega_{2} / \omega_{1}\right)$.

The derivative of $\zeta$ is $-\wp$. From

$$
\wp^{\prime \prime}=6 \wp^{2}-\left(g_{2} / 2\right)
$$

one deduces that $s_{k}(\Omega)$ is a homogeneous polynomial in $\mathbb{Q}\left[g_{2}, g_{3}\right]$ of weight $2 k$ for the graduation of $\mathbb{Q}\left[g_{2}, g_{3}\right]$ determined by assigning to $g_{2}$ the degree 4 and to $g_{3}$ the degree 6.

As a side remark, we notice that for any $u \in \mathbb{C} \backslash \Omega$ we have

$$
\mathbb{Q}\left(g_{2}, g_{3}\right) \subset \mathbb{Q}\left(\wp(u), \wp^{\prime}(u), \wp^{\prime \prime}(u)\right) .
$$

Since its derivative is periodic, the function $\zeta$ is quasiperiodic: for each $\omega \in \Omega$ there is a complex number $\eta=\eta(\omega)$ such that

$$
\zeta(z+\omega)=\zeta(z)+\eta
$$

These numbers $\eta$ are the quasiperiods of the elliptic curve. If $\left(\omega_{1}, \omega_{2}\right)$ is a pair of fundamental periods and if we set $\eta_{1}=\eta\left(\omega_{1}\right)$ and $\eta_{2}=\eta\left(\omega_{2}\right)$, then, for $(a, b) \in \mathbb{Z}^{2}$,

$$
\eta\left(a \omega_{1}+b \omega_{2}\right)=a \eta_{1}+b \eta_{2}
$$


Returning to the sigma function, one deduces that

$$
\sigma\left(z+\omega_{i}\right)=-\sigma(z) \exp \left(\eta_{i}\left(z+\left(\omega_{i} / 2\right)\right)\right) \quad(i=1,2) .
$$

The zeta function also satisfies an addition formula:

$$
\zeta\left(z_{1}+z_{2}\right)=\zeta\left(z_{1}\right)+\zeta\left(z_{2}\right)+\frac{1}{2} \cdot \frac{\wp^{\prime}\left(z_{1}\right)-\wp^{\prime}\left(z_{2}\right)}{\wp\left(z_{1}\right)-\wp\left(z_{2}\right)} .
$$

The Legendre relation relating the periods and the quasiperiods

$$
\omega_{2} \eta_{1}-\omega_{1} \eta_{2}=2 \pi i
$$

when $\omega_{2} / \omega_{1}$ has positive imaginary part, can be obtained by integrating $\zeta(z)$ along the boundary of a fundamental parallelogram ([43] Section 4.2, [124] Section 1.6, [244] Section 20.411).

In the case of complex multiplication, if $\tau$ is the quotient of a pair of fundamental periods of $\wp$, then the function $\zeta(\tau z)$ is algebraic over the field $\mathbb{Q}\left(g_{2}, g_{3}, z, \wp(z)\right.$, $\zeta(z))$.

Examples $([4,241])$. For the curve $y^{2} t=4 x^{3}-4 x t^{2}$, the quasiperiods attached to the above-mentioned pair of fundamental periods (5) are

$$
\eta_{1}=\frac{\pi}{\omega_{1}}=\frac{(2 \pi)^{3 / 2}}{\Gamma(1 / 4)^{2}}, \quad \eta_{2}=-i \eta_{1}
$$

it follows that the fields $\mathbb{Q}\left(\omega_{1}, \omega_{2}, \eta_{1}, \eta_{2}\right)$ and $\mathbb{Q}(\pi, \Gamma(1 / 4))$ have the same algebraic closure over $\mathbb{Q}$, hence the same transcendence degree. For the curve $y^{2} t=4 x^{3}-4 t^{3}$ with periods (6), they are

$$
\eta_{1}=\frac{2 \pi}{\sqrt{3} \omega_{1}}=\frac{2^{7 / 3} \pi^{2}}{3^{1 / 2} \Gamma(1 / 3)^{3}}, \quad \eta_{2}=\varrho^{2} \eta_{1} .
$$

In this case the fields $\mathbb{Q}\left(\omega_{1}, \omega_{2}, \eta_{1}, \eta_{2}\right)$ and $\mathbb{Q}(\pi, \Gamma(1 / 3))$ have the same algebraic closure over $\mathbb{Q}$, hence the same transcendence degree.

\subsection{Elliptic Integrals}

Let

$$
\mathcal{E}=\left\{(t: x: y) \in \mathbb{P}_{2} ; y^{2} t=4 x^{3}-g_{2} x t^{2}-g_{3} t^{3}\right\}
$$

be an elliptic curve. The field of rational (meromorphic) functions on $\mathcal{E}$ over $\mathbb{C}$ is $\mathbb{C}(\mathcal{E})=\mathbb{C}\left(\wp, \wp^{\prime}\right)=\mathbb{C}(x, y)$, where $x$ and $y$ are related by the cubic equation $y^{2}=4 x^{3}-g_{2} x-g_{3}$. Under the isomorphism $\mathbb{C} / \Omega \rightarrow \mathcal{E}(\mathbb{C})$ given by $\left(1: \wp: \wp^{\prime}\right)$, the differential form $d z$ is mapped to $d x / y$. The holomorphic differential forms on $\mathbb{C} / \Omega$ are $\lambda d z$ with $\lambda \in \mathbb{C}$. 
The differential form $d \zeta=\zeta^{\prime} / \zeta$ is mapped to $-x d x / y$. The differential forms of the second kind on $\mathcal{E}(\mathbb{C})$ are $a d z+b d \zeta+d \chi$, where $a$ and $b$ are complex numbers and $\chi \in \mathbb{C}(x, y)$ is a meromorphic function on $\mathcal{E}$.

Assume that the elliptic curve $\mathcal{E}$ is defined over $\overline{\mathbb{Q}}$ : the invariants $g_{2}$ and $g_{3}$ are algebraic. We shall be interested in differential forms defined over $\overline{\mathbb{Q}}$. Those of the second kind are $a d z+b d \zeta+d \chi$, where $a$ and $b$ are algebraic numbers and $\chi \in$ $\overline{\mathbb{Q}}(x, y)$.

An elliptic integral (see [244] Section 22.7; see also [43] Section 1.4 and [212] Section 6.1) is an integral

$$
\int R(x, y) d x
$$

where $R$ is a rational function of $x$ and $y$, while $y^{2}$ is a polynomial in $x$ of degree 3 or 4 without multiple roots, with the proviso that the integral cannot be integrated by means of elementary functions. One may transform this integral as follows: one reduces it to an integral of $d x / \sqrt{P(x)}$, where $P$ is a polynomial of third or fourth degree; in case $P$ has degree 4 , one replaces it with a degree- 3 polynomial by sending one root to infinity; finally, one reduces it to a Weierstrass equation by means of a birational transformation. The value of the integral is not modified.

For transcendence purposes, if the initial differential form is defined over $\overline{\mathbb{Q}}$, then all these transformations involve only algebraic numbers.

We refer to Section 22.7 of [244] for the definition of elliptic integrals of the first, second, and third kinds.

\section{Transcendence Results of Numbers Related to Elliptic Functions}

\subsection{Elliptic Analogue of Lindemann's Theorem on the Transcendence of $\pi$ and the Hermite-Lindemann Theorem on the Transcendence of $\log \alpha$}

The first transcendence result on periods of elliptic functions was proved by C.L. Siegel [210] as early as 1932.

Theorem 17 (Siegel, 1932). Let $\wp$ be a Weierstrass elliptic function with period lattice $\mathbb{Z} \omega_{1}+\mathbb{Z} \omega_{2}$. Assume that the invariants $g_{2}$ and $g_{3}$ of $\wp$ are algebraic. Then at least one of the two numbers $\omega_{1}, \omega_{2}$ is transcendental.

One main feature of Siegel's proof is that he used Dirichlet's box principle (the so-called Thue-Siegel lemma, which is included in his 1929 paper) to construct an auxiliary function. This idea turned out to be of fundamental importance for the solution of Hilbert's seventh problem by Gel'fond and Schneider two years later.

In the case of complex multiplication, it follows from Theorem 17 that any nonzero period of $\wp$ is transcendental.

From formulas (5) and (6) it follows as a consequence of Siegel's 1932 result [210] that both numbers $\Gamma(1 / 4)^{4} / \pi$ and $\Gamma(1 / 3)^{3} / \pi$ are transcendental. 
Other consequences of Siegel's result concern the transcendence of the length of an arc of an ellipse [207, 211]:

$$
2 \int_{-b}^{b} \sqrt{1+\frac{a^{2} x^{2}}{b^{4}-b^{2} x^{2}}} d x
$$

for algebraic $a$ and $b$, as well as the transcendence of an arc of the lemniscate $\left(x^{2}+y^{2}\right)^{2}=2 a^{2}\left(x^{2}-y^{2}\right)$ with $a$ algebraic.

A further example of application of Siegel's theorem [211] is the transcendence of values of hypergeometric series related to elliptic integrals

$$
K(z)=\int_{0}^{1} \frac{d x}{\sqrt{\left(1-x^{2}\right)\left(1-z^{2} x^{2}\right)}}=\frac{\pi}{2} \cdot{ }_{2} F_{1}\left(1 / 2,1 / 2 ; 1 \mid z^{2}\right),
$$

where ${ }_{2} F_{1}$ denotes the Gauss hypergeometric series

$$
{ }_{2} F_{1}(a, b ; c \mid z)=\sum_{n=0}^{\infty} \frac{(a)_{n}(b)_{n}}{(c)_{n}} \cdot \frac{z^{n}}{n !}
$$

with $(a)_{n}=a(a+1) \cdots(a+n-1)$.

Further results on this topic were obtained by Th. Schneider [203] in 1934 and in joint work by K. Mahler and J. Popken [190] in 1935 using Siegel's method. These results were superseded by Th. Schneider's fundamental memoir [204] in 1936 in which he proved a number of definitive results on the subject, including the following:

Theorem 18 (Schneider, 1936). Assume that the invariants $g_{2}$ and $g_{3}$ of $\wp$ are algebraic. Then for any nonzero period $\omega$ of $\wp$, the numbers $\omega$ and $\eta(\omega)$ are transcendental.

It follows from Theorem 18 that any nonzero period of an elliptic integral of the first or second kind is transcendental:

Corollary 19. Let $\mathcal{E}$ be an elliptic curve over $\overline{\mathbb{Q}}, p_{1}$ and $p_{2}$ two algebraic points on $\mathcal{E}(\overline{\mathbb{Q}}), w$ a differential form of the first or second kind on $\mathcal{E}$ that is defined over $\overline{\mathbb{Q}}$, holomorphic at $p_{1}$ and $p_{2}$, and is not the differential of a rational function. Let $\gamma$ be a path on $\mathcal{E}$ from $p_{1}$ to $p_{2}$. In case $p_{1}=p_{2}$ one assumes that $\gamma$ is not homologous to 0 . Then the number

$$
\int_{\gamma} w
$$

is transcendental.

Examples. Using Corollary 19 and formulas (9) and (10), one deduces that the numbers

$$
\Gamma(1 / 4)^{4} / \pi^{3} \text { and } \Gamma(1 / 3)^{3} / \pi^{2}
$$

are transcendental. 
The main results of Schneider's 1936 paper [204] are as follows (see also [207]):

\section{Theorem 20 (Schneider, 1936).}

1. Let $\wp$ be a Weierstrass elliptic function with algebraic invariants $g_{2}, g_{3}$. Let $\beta$ be a nonzero algebraic number. Then $\beta$ is not a pole of $\wp$ and $\wp(\beta)$ is transcendental. More generally, if $a$ and $b$ are two algebraic numbers with $(a, b) \neq(0,0)$, then for any $u \in \mathbb{C} \backslash \Omega$ at least one of the two numbers $\wp(u), a u+b \zeta(u)$ is transcendental.

2. Let $\wp$ and $\wp^{*}$ be two algebraically independent elliptic functions with algebraic invariants $g_{2}, g_{3}, g_{2}^{*}, g_{3}^{*}$. If $t \in \mathbb{C}$ is not a pole of $\wp$ or of $\wp^{*}$, then at least one of the two numbers $\wp(t)$ and $\wp^{*}(t)$ is transcendental.

3. Let $\wp$ be a Weierstrass elliptic function with algebraic invariants $g_{2}, g_{3}$. Then for any $t \in \mathbb{C} \backslash \Omega$, at least one of the two numbers $\wp(t)$, $e^{t}$ is transcendental.

It follows from Theorem 20.2 that the quotient of an elliptic integral of the first kind (between algebraic points) by a nonzero period is either in the field of endomorphisms (hence a rational number, or a quadratic number in the field of complex multiplication), or a transcendental number.

Here is another important consequence of Theorem 20.2.

Corollary 21 (Schneider, 1936). Let $\tau \in \mathcal{H}$ be a complex number in the upper half-

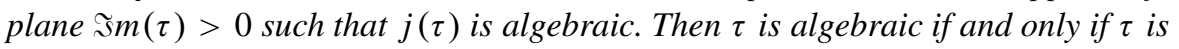
imaginary quadratic.

In this connection we quote Schneider's second problem in [207], which is still open (see Wakabayashi's papers [226, 227, 228]):

Open Problem. Prove Corollary 21 without using elliptic functions.

Sketch of proof of Corollary 21 as a consequence of part 2 of Theorem 20. Assume that both $\tau \in \mathcal{H}$ and $j(\tau)$ are algebraic. There exists an elliptic function with algebraic invariants $g_{2}, g_{3}$ and periods $\omega_{1}, \omega_{2}$ such that

$$
\tau=\frac{\omega_{2}}{\omega_{1}} \quad \text { and } \quad j(\tau)=\frac{1728 g_{2}^{3}}{g_{2}^{3}-27 g_{3}^{2}} .
$$

Set $\wp^{*}(z)=\tau^{2} \wp(\tau z)$. Then $\wp^{*}$ is a Weierstrass function with algebraic invariants $g_{2}^{*}, g_{3}^{*}$. For $u=\omega_{1} / 2$ the two numbers $\wp(u)$ and $\wp^{*}(u)$ are algebraic. Hence the two functions $\wp(z)$ and $\wp^{*}(z)$ are algebraically dependent. It follows that the corresponding elliptic curve has nontrivial endomorphisms; therefore $\tau$ is quadratic.

A quantitative refinement of Schneider's theorem on the transcendence of $j(\tau)$ given by A. Faisant and G. Philibert in 1984 [74] became useful 10 years later in connection with Nesterenko's result (see Section 5). See also [75].

We will not review the results related to abelian integrals, but only quote the first result on this topic, which involves the Jacobian of a Fermat curve: in 1941 Schneider [205] proved that for $a$ and $b$ in $\mathbb{Q}$ with $a, b$ and $a+b$ not in $\mathbb{Z}$, the number 


$$
B(a, b)=\frac{\Gamma(a) \Gamma(b)}{\Gamma(a+b)}
$$

is transcendental. We notice that in his 1932 paper [210], C.L. Siegel had already announced partial results on the values of the Euler gamma function (see also [19]).

Schneider's above-mentioned results deal with elliptic (and abelian) integrals of the first or second kind. His method can be extended to deal with elliptic (and abelian) integrals of the third kind (this is Schneider's third problem in [207]).

As pointed out by J.-P. Serre in 1979 [233], it follows from the quasiperiodicity of the Weierstrass sigma function (8) that the function

$$
F_{u}(z)=\frac{\sigma(z+u)}{\sigma(z) \sigma(u)} e^{-z \zeta(u)}
$$

satisfies

$$
F_{u}\left(z+\omega_{i}\right)=F_{u}(z) e^{\eta_{i} u-\omega_{i} \zeta(u)} .
$$

Theorem 22. Let $u_{1}$ and $u_{2}$ be two nonzero complex numbers. Assume that $g_{2}, g_{3}$, $\wp\left(u_{1}\right), \wp\left(u_{2}\right), \beta$ are algebraic and $\mathbb{Z} u_{1} \cap \Omega=\{0\}$. Then the number

$$
\frac{\sigma\left(u_{1}+u_{2}\right)}{\sigma\left(u_{1}\right) \sigma\left(u_{2}\right)} e^{\left(\beta-\zeta\left(u_{1}\right)\right) u_{2}}
$$

is transcendental.

From the next corollary, one can deduce that nonzero periods of elliptic integrals of the third kind are transcendental (see [232]).

Corollary 23. For any nonzero period $\omega$ and for any $u \in \mathbb{C} \backslash \Omega$, the number $e^{\omega \zeta(u)-\eta u+\beta \omega}$ is transcendental.

Further results on elliptic integrals are due to M. Laurent [132]. See also his papers [134, 135, 136, 137].

Ya. M. Kholyavka wrote several papers devoted to the approximation of transcendental numbers related to elliptic functions [106, 107, 108, 109, 110, 111, 112, $113,114]$.

Quantitative estimates (measures of transcendence) related to the results of this section were derived by N.I. Fel'dman [76, 77, 78, 79, 80]. See also the papers by S. Lang [119], N.D. Nagaev [165], N. Hirata [101], E. Reyssat [195, 196, 198, 199], M. Laurent [133], R. Tubbs [219], G. Diaz [64], N. Saradha [202], P. Grinspan [94].

\subsection{Elliptic Analogues of the Six Exponentials Theorem}

Elliptic analogs of the six exponentials theorem (Theorem 10) were considered by S. Lang [120] and K. Ramachandra [191] in the 1960s.

Let $d_{1}, d_{2}$ be nonnegative integers and $m$ a positive integer, let $x_{1}, \ldots, x_{d_{1}}$ be complex numbers that are linearly independent over $\mathbb{Q}$, let $y_{1}, \ldots, y_{m}$ be complex numbers that are linearly independent over $\mathbb{Q}$, and let $u_{1}, \ldots, u_{d_{2}}$ be nonzero 
complex numbers. We consider Weierstrass elliptic functions $\wp_{1}, \ldots, \wp_{d_{2}}$ and we denote by $K_{0}$ the field generated over $\mathbb{Q}$ by their invariants $g_{2, k}$ and $g_{3, k}(1 \leq$ $\left.k \leq d_{2}\right)$. We assume that the $d_{2}$ functions $\wp_{1}\left(u_{1} z\right), \ldots, \wp_{d_{2}}\left(u_{d_{2}} z\right)$ are algebraically independent. We denote by $K_{1}$ the field generated over $K_{0}$ by the numbers $\exp \left(x_{i} y_{j}\right)$ $\left(1 \leq i \leq d_{1}, 1 \leq j \leq m\right)$ together with the numbers $\wp_{k}\left(u_{k} y_{j}\right)\left(1 \leq k \leq d_{2}\right.$, $1 \leq j \leq m)$. Next, define

$$
K_{2}=K_{1}\left(y_{1}, \ldots, y_{m}\right), \quad K_{3}=K_{1}\left(x_{1}, \ldots, x_{d_{1}}, u_{1}, \ldots, u_{d_{2}}\right),
$$

and let $K_{4}$ be the compositum of $K_{2}$ and $K_{3}$ :

$$
K_{4}=K_{1}\left(y_{1}, \ldots, y_{m}, x_{1}, \ldots, x_{d_{1}}, u_{1}, \ldots, u_{d_{2}}\right) .
$$

The theorems of Hermite-Lindemann (Theorem 3), Gel'fond-Schneider (Theorem 7), the six exponentials theorem, and their elliptic analogues due to Schneider, Lang, and Ramachandra can be stated as follows.

Any one of the four assumptions below will imply $d_{1}+d_{2}>0$; the case in which $d_{1}$ (respectively $d_{2}$ ) vanishes means that one considers only elliptic (respectively exponential) functions.

\section{Theorem 24.}

1. Assume $\left(d_{1}+d_{2}\right) m>m+d_{1}+2 d_{2}$. Then the field $K_{1}$ has transcendence degree $\geq 1$ over $\mathbb{Q}$.

2. Assume either $d_{1} \geq 1$ and $m \geq 2$, or $d_{2} \geq 1$ and $m \geq 3$. Then $K_{2}$ has transcendence degree $\geq 1$ over $\mathbb{Q}$.

3. Assume $d_{1}+\bar{d}_{2} \geq 2$. Then $K_{3}$ has transcendence degree $\geq 1$ over $\mathbb{Q}$.

4. Assume $d_{1}+d_{2} \geq 1$. Then $K_{4}$ has transcendence degree $\geq 1$ over $\mathbb{Q}$.

Parts 3 and 4 of Theorem 24 are consequences of the Schneider-Lang criterion [120], which deals with meromorphic functions satisfying differential equations, while parts 1 and 2 follow from a criterion that involves no differential equations. Such criteria were given by Schneider [206, 207], Lang [120], and Ramachandra [191] (see also [228] and [229]).

Theorem 24 also includes Theorem 20 apart from the case $b \neq 0$ in part 1 of that statement. However, there are extensions of Theorem 24 that include results on Weierstrass zeta functions (and also on Weierstrass sigma functions in connection with elliptic integrals of the third kind). See [132, 134, 135, 136, 137, 199, 232, 233].

Here is a corollary of part 1 of Theorem 24 (take $d_{1}=0, d_{2}=3, \wp_{1}=\wp_{2}=$ $\wp_{3}=\wp, m=4, y_{1}=1, y_{2} \in \operatorname{End}(E) \backslash \mathbb{Q}, y_{3}=v_{1} / u_{1}, y_{4}=y_{2} y_{3}$; there is an alternative proof with $d_{2}=2$ and $m=6$ ).

Corollary 25. Let $E$ be an elliptic curve with algebraic invariants $g_{2}, g_{3}$. Assume that $E$ has complex multiplication. Let

$$
M=\left(\begin{array}{lll}
u_{1} & u_{2} & u_{3} \\
v_{1} & v_{2} & v_{3}
\end{array}\right)
$$


be a $2 \times 3$ matrix whose entries are elliptic logarithms of algebraic numbers, i.e., $u_{i}$ and $v_{i}(i=1,2,3)$ are in $\mathcal{L}_{E}$. Assume that the three columns are linearly independent over $\operatorname{End}(E)$ and the two rows are also linearly independent over $\operatorname{End}(E)$. Then the matrix $M$ has rank 2.

In the non-CM case, one deduces from Theorem 24 a similar (but weaker) statement according to which such matrices $\left(u_{i j}\right)$ (where $\wp\left(u_{i j}\right)$ are algebraic numbers) have rank $\geq 2$ if they have size $2 \times 5$ (taking $d_{1}=0, d_{2}=2$, and $m=5$ ) or $3 \times 4$ (taking $d_{1}=0$ and either $d_{2}=3, m=4$ or $d_{2}=4$ and $m=3$ ) instead of $2 \times 3$.

Lower bounds better than 2 for the rank of matrices of larger sizes are known, but we will not discuss this question here. We just mention the fact that higherdimensional considerations are relevant to a problem discussed by B. Mazur on the density of rational points on varieties [240].

\section{Linear Independence of Numbers Related to Elliptic Functions}

From Schneider's theorem (Theorem 20) part 1, one deduces the linear independence over the field of algebraic numbers of the three numbers $1, \omega$, and $\eta$, when $\omega$ is a nonzero period of a Weierstrass elliptic function (with algebraic invariants $g_{2}$ and $\left.g_{3}\right)$ and $\eta=\eta(\omega)$ is the associated quasiperiod of the corresponding Weierstrass zeta function. However, the Gel'fond-Schneider method in one variable alone does not yield strong results of linear independence. Baker's method is better suited for this purpose.

\subsection{Linear Independence of Periods and Quasiperiods}

Baker's method of proof for his theorem (Theorem 9) on linear independence of logarithms of algebraic numbers was used as early as 1969 and 1970 by A. Baker himself $[12,10]$ when he proved the transcendence of linear combinations with algebraic coefficients of the numbers $\omega_{1}, \omega_{2}, \eta_{1}$, and $\eta_{2}$ associated with an elliptic curve having algebraic invariants $g_{2}$ and $g_{3}$. His method is effective: it provides quantitative Diophantine estimates [11].

In 1971, J. Coates [52] proved the transcendence of linear combinations with algebraic coefficients of $\omega_{1}, \omega_{2}, \eta_{1}, \eta_{2}$, and $2 \pi i$. Moreover, he proved in [51, 53, $54,55]$ that in the non-CM case, the three numbers $\omega_{1}, \omega_{2}$, and $2 \pi i$ are $\overline{\mathbb{Q}}$-linearly independent. Further results including usual logarithms of algebraic numbers are due to T. Harase in 1974 and 1976 [97, 98].

The final result on the question of linear dependence of periods and quasiperiods for a single elliptic function was given by D.W. Masser in 1975 [143, 144].

Theorem 26 (Masser, 1975). Let §o be a Weierstrass elliptic function with algebraic invariants $g_{2}$ and $g_{3}$, denote by $\zeta$ the corresponding Weierstrass zeta function, let $\omega_{1}, \omega_{2}$ be a basis of the period lattice of $\wp$, and let $\eta_{1}, \eta_{2}$ be the associated quasiperiods of $\zeta$. Then the six numbers $1, \omega_{1}, \omega_{2}, \eta_{1}, \eta_{2}$, and $2 \pi i$ span a $\overline{\mathbb{Q}}$-vector space of dimension 6 in the non-CM case, 4 in the CM case: 


$$
\operatorname{dim}_{\overline{\mathbb{Q}}}\left\{1, \omega_{1}, \omega_{2}, \eta_{1}, \eta_{2}, 2 \pi i\right\}=2+2 \operatorname{dim}_{\overline{\mathbb{Q}}}\left\{\omega_{1}, \omega_{2}\right\} .
$$

The fact that the dimension is 4 in the CM case means that there are two independent linear relations among these six numbers. One of them is $\omega_{2}=\tau \omega_{1}$ with $\tau \in \overline{\mathbb{Q}}$. The second one (see [144]; see also [37]) can be written

$$
C^{2} \tau \eta_{2}-A C \eta_{1}+\gamma \omega_{1}=0
$$

where $A+B X+C X^{2}$ is the minimal polynomial of $\tau$ over $\mathbb{Z}$ and $\gamma$ is an element in $\mathbb{Q}\left(g_{2}, g_{3}, \tau\right)$.

In [144], D.W. Masser also produced quantitative estimates (measures of linear independence). In 1976, R. Franklin and D.W. Masser [85, 151] obtained an extension involving a logarithm of an algebraic number.

Further results can be found in papers by P. Bundschuh [40], S. Lang's surveys [121, 122], D.W. Masser [152, 154], M. Anderson [5], and in the joint paper [6] by M. Anderson and D.W. Masser.

\subsection{Elliptic Analogue of Baker's Theorem}

The elliptic analogue of Baker's theorem on linear independence of logarithms was proved by D.W. Masser in 1974 [143, 144] in the CM case.

His proof also yields quantitative estimates (measures of linear independence of elliptic logarithms of algebraic points on an elliptic curve). Such estimates have a number of applications: this was shown by A.O. Gel'fond for usual logarithms of algebraic numbers [89], and further consequences of such lower bounds in the case of elliptic curves for solving Diophantine equations (integer points on elliptic curves) were derived by S. Lang [126].

Lower bounds for linear combinations of elliptic logarithms in the CM case were obtained by several mathematicians including J. Coates [52], D.W. Masser [145, 149, 150], J. Coates and S. Lang [56], M. Anderson [5]. The work of Yu Kunrui [253] yields similar estimates, but his method is not that of Baker-Masser: instead of using a generalization of Gel'fand's solution to Hilbert's seventh problem, Yu Kunrui uses a generalization in several variables of Schneider's solution to the same problem. Again, this method is restricted to the CM case.

The question of linear independence of elliptic logarithms in the non-CM case was settled only in 1980 by D. Bertrand and D.W. Masser [30, 31]. They found a new proof of Baker's theorem using functions of several variables, and they were able to extend this argument to the situation of elliptic functions, either with or without complex multiplication. The criterion they use is the one that Schneider established in 1949 [205] for his proof of the transcendence of beta values. This criterion (revisited by S. Lang in [120]) deals with Cartesian products. From the several variables point of view, this is a rather degenerate situation; much deeper results are available, including Bombieri's solution in 1970 of Nagata's conjecture [120, 233], which involves Hörmander $L^{2}$-estimates for analytic functions of several variables. However Bombieri's theorem does not seem to yield new transcendence results, so far. 
So far, these deeper results do not give further transcendence results in our context.

Theorem 27 (D.W. Masser 1974 for the CM case, D. Bertrand and D.W. Masser 1980 for the non-CM case). Let $\wp$ be a Weierstrass elliptic function with algebraic invariants $g_{2}, g_{3}$ and field of endomorphisms $k$. Let $u_{1}, \ldots, u_{n}$ be $k$-linearly independent complex numbers. Assume, for $1 \leq i \leq n$, that either $u_{i} \in \Omega$ or $\wp\left(u_{i}\right) \in \overline{\mathbb{Q}}$. Then the numbers $1, u_{1}, \ldots, u_{n}$ are linearly independent over the field $\overline{\mathbb{Q}}$.

This means that for an elliptic curve $E$ that is defined over $\overline{\mathbb{Q}}$, if $u_{1}, \ldots, u_{n}$ are elements in $\mathcal{L}_{E}$ that are linearly independent over the field of endomorphisms of $E$, then the numbers $1, u_{1}, \ldots, u_{n}$ are linearly independent over $\overline{\mathbb{Q}}$.

The method of Bertrand-Masser yields only weak Diophantine estimates (measures of linear independence of logarithms).

\subsection{Further Results of Linear Independence}

Theorem 26 deals only with periods and quasiperiods associated with one lattice; Theorem 27 deals only with elliptic logarithms of algebraic points on one elliptic curve. A far-reaching generalization of both results was achieved by G. Wüstholz in 1987 [249, 250, 251] when he succeeded in extending Baker's theorem to abelian varieties and integrals, and, more generally, to commutative algebraic groups. If we restrict his general result to products of a commutative linear group, of copies of elliptic curves, and of extensions of elliptic curves by the additive or the multiplicative group, the resulting statement settles the questions of linear independence of logarithms of algebraic numbers and of elliptic logarithms of algebraic points, including periods, quasiperiods, elliptic integrals of the first, second, or third kind. This is a main step toward an answer to the questions of M. Kontsevich and D. Zagier on periods [118].

Wüstholz's method can be extended to yield measures of linear independence of logarithms of algebraic points on an algebraic group. The first effective such lower bounds were given in 1989 [188, 189]. As a special case, they provide the first measures of linear independence for elliptic logarithms that is also valid in the non-CM case. More generally, they give effective lower bounds for any nonvanishing linear combination of logarithms of algebraic points on algebraic groups (including usual logarithms, elliptic logarithms, elliptic integrals of any kind).

Refinements were obtained by N. Hirata-Kohno [100, 101, 102, 103, 104], S. David [60], N. Hirata-Kohno and S. David [62], M. Ably [2, 3], and É. Gaudron $[86,87,88]$, who uses not only Hirata's reduction argument, but also the work of J-B. Bost [33] (slope inequalities) involving Arakelov's theory. For instance, thanks to the recent work of David and Hirata-Kohno on the one hand, of Gaudron on the other, one knows that the above-mentioned nonvanishing linear combinations of logarithms of algebraic points are not Liouville numbers.

In the $p$-adic case there is a paper of G. Rémond and F. Urfels [194] dealing with two elliptic logarithms. 
Further applications to elliptic curves of the Baker-Masser-Wüstholz method were derived by D.W. Masser and G. Wüstholz [163, 164].

A survey on questions related to the isogeny theorem is [178]. Other surveys dealing with the questions of small points, Bogomolov conjecture, and the André Oort conjecture are $[59,61]$. We do not cover these aspects of the theory in the present paper. Other related topics that would deserve more attention are the theory of height and theta functions as well as ultrametric questions.

Extensions of the above-mentioned results to abelian varieties were considered by D.W. Masser [145, 146, 147, 148, 149, 150, 153, 155, 156, 157], S. Lang [123], J. Coates and S. Lang [56], D. Bertrand and Y.Z. Flicker [28], Y.Z. Flicker [84], D. Bertrand [25, 26]. For instance, J. Wolfart and G. Wüstholz [245] have shown that the only linear dependence relations with algebraic coefficients between the values $B(a, b)$ of the Euler beta function at points $(a, b) \in \mathbb{Q}^{2}$ are those that follow from the Deligne-Koblitz-Ogus relations (see further references in [243]).

\section{Algebraic Independence of Numbers Related to Elliptic Functions}

\subsection{Small Transcendence Degree}

We keep the notation and assumptions of Section 3.2.

The following extension of Theorem 24 to a result of algebraic independence containing Gel'fond's 1948 results on the exponential function (see Section 1.1) is a consequence of the work of many mathematicians, including A.O. Gel'fond [89], A.A. S̆melev [214, 215], W.D. Brownawell [35], W.D. Brownawell and K.K. Kubota [37], G. Wüstholz [246], D.W. Masser and G. Wüstholz [160], and others (further references are given in $[35,235,236])$.

\section{Theorem 28.}

1. Assume $\left(d_{1}+d_{2}\right) m \geq 2\left(m+d_{1}+2 d_{2}\right)$. Then the field $K_{1}$ has transcendence degree $\geq 2$ over $\mathbb{Q}$.

2. Assume $\left(d_{1}+d_{2}\right) m \geq m+2\left(d_{1}+2 d_{2}\right)$. Then $K_{2}$ has transcendence degree $\geq 2$ over $\mathbb{Q}$.

3. Assume $\left(d_{1}+d_{2}\right) m \geq 2 m+d_{1}+2 d_{2}$. Then $K_{3}$ has transcendence degree $\geq 2$ over $\mathbb{Q}$.

4. Assume $\left(d_{1}+d_{2}\right) m>m+d_{1}+2 d_{2}$. Then $K_{4}$ has transcendence degree $\geq 2$ over $\mathbb{Q}$.

Quantitative estimates (measures of algebraic independence) exist (R. Tubbs [220] and E.M. Jabbouri [105]).

Further related results are due to N.I. Fel'dman [81, 82], R. Tubbs [219, 220, 221, 222, 223, 224], É. Reyssat [201], M. Toyoda and T. Yasuda [218]. See also the measure of algebraic independence given by M. Ably in [1] and by S.O. Shestakov in [209]. 
A survey on results related to small transcendence degree is given in [236] (see also Chapter 13 of [177]).

Again, as for Theorem 24, there are extensions of Theorem 28 that include results on Weierstrass zeta functions as well as on functions of several variables, with a number of consequences related to abelian functions [237].

\subsection{Algebraic Independence of Periods and Quasiperiods}

In the 1970s, G.V. Chudnovsky proved strong results of algebraic independence (small transcendence degree) related to elliptic functions. One of his most spectacular contributions was obtained in 1976 [45] (see also [48] and [50]):

Theorem 29 (G.V. Chudnovsky, 1976). Let $\wp$ be a Weierstrass elliptic function with invariants $g_{2}, g_{3}$. Let $\left(\omega_{1}, \omega_{2}\right)$ be a basis of the lattice period of $\wp$ and $\eta_{1}=\eta\left(\omega_{1}\right)$, $\eta_{2}=\eta\left(\omega_{2}\right)$ the associated quasiperiods of the associated Weierstrass zeta function. Then at least two of the numbers $g_{2}, g_{3}, \omega_{1}, \omega_{2}, \eta_{1}, \eta_{2}$ are algebraically independent.

A more precise result ([50] Chapter 7, Theorem 3.1) is that for any nonzero period $\omega$, at least two of the four numbers $g_{2}, g_{3}, \omega / \pi, \eta / \omega$ (with $\eta=\eta(\omega)$ ) are algebraically independent.

In the case that $g_{2}$ and $g_{3}$ are algebraic, one deduces from Theorem 29 that two among the four numbers $\omega_{1}, \omega_{2}, \eta_{1}, \eta_{2}$ are algebraically independent; this statement is also a consequence of the next result (Theorem 4 of [48]; see also [50, 235]):

Theorem 30 (G.V. Chudnovsky, 1981). Assume that $g_{2}$ and $g_{3}$ are algebraic. Let $\omega$ be a nonzero period of $\wp$, set $\eta=\eta(\omega)$, and let $u$ be a complex number that is not a period such that $u$ and $\omega$ are $\mathbb{Q}$-linearly independent: $u \notin \mathbb{Q} \omega \cup \Omega$. Assume $\wp(u) \in \overline{\mathbb{Q}}$. Then the two numbers

$$
\zeta(u)-\frac{\eta}{\omega} u, \quad \frac{\eta}{\omega}
$$

are algebraically independent.

From Theorem 29 or Theorem 30 one deduces the following result:

Corollary 31. Let $\omega$ be a nonzero period of $\wp$ and $\eta=\eta(\omega)$. If $g_{2}$ and $g_{3}$ are algebraic, then the two numbers $\pi / \omega$ and $\eta / \omega$ are algebraically independent.

The following consequence of Corollary 31 shows that in the CM case, Chudnovsky's results are sharp:

Corollary 32. Assume that $g_{2}$ and $g_{3}$ are algebraic and the elliptic curve has complex multiplication. Let $\omega$ be a nonzero period of $\wp$. Then the two numbers $\omega$ and $\pi$ are algebraically independent. 
As a consequence of formulas (5) and (6), one deduces the following corollary:

Corollary 33. The numbers $\pi$ and $\Gamma(1 / 4)$ are algebraically independent. Also the numbers $\pi$ and $\Gamma(1 / 3)$ are algebraically independent.

In connection with these results let us quote a conjecture of S. Lang from 1971 [121] p. 652.

Conjecture 34. If $j(\tau)$ is algebraic with $j^{\prime}(\tau) \neq 0$, then $j^{\prime}(\tau)$ is transcendental.

According to Siegel's relation (see [121] p. 652 and [66] Section 1.2.5 p. 165),

$$
j^{\prime}(\tau)=18 \frac{\omega_{1}^{2}}{2 \pi i} \cdot \frac{g_{3}}{g_{2}} \cdot j(\tau) .
$$

Conjecture 34 amounts to the transcendence of $\omega^{2} / \pi$. Hence Corollary 32 implies that Conjecture 34 is true at least in the CM case (see [22]):

Corollary 35. If $\tau \in \mathcal{H}$ is quadratic and $j^{\prime}(\tau) \neq 0$, then $\pi$ and $j^{\prime}(\tau)$ are algebraically independent.

A quantitative refinement (measure of algebraic independence) of Corollary 31 due to G. Philibert [181] turns out to be useful in connection with Nesterenko's work in 1996 (further references on this topic are given in [239]).

A transcendence measure for $\Gamma(1 / 4)$ was obtained by P. Philippon $[186,187]$ and S. Bruiltet [39]:

Theorem 36. For $P \in \mathbb{Z}[X, Y]$ with degree $d$ and height $H$,

$$
\log \mid P\left(\pi, \Gamma(1 / 4) \mid>-10^{326}\left((\log H+d \log (d+1)) d^{2}(\log (d+1))^{2} .\right.\right.
$$

Corollary 37. The number $\Gamma(1 / 4)$ is not a Liouville number:

$$
\left|\Gamma(1 / 4)-\frac{p}{q}\right|>\frac{1}{q^{10^{330}}} .
$$

Further algebraic independence results can be found in papers including those of D. Bertrand [20, 23], G.V. Chudnovsky [49] (however, see Zbl 0456.10016) and E. Reyssat [197, 200] (see also the Bourbaki lecture [231] and the book of E.B. Burger and R. Tubbs [42]). Among Chudnovsky's other contributions are results dealing with $G$-functions (see [50]; see also Y. André's work [7, 8]).

We conclude this section with the following open problem, which simultaneously generalizes Theorems 29 and 30 of G.V. Chudnovsky.

Conjecture 38. Let $\wp$ be a Weierstrass elliptic function with invariants $g_{2}, g_{3}$, let $\omega$ be a nonzero period of $\wp$, set $\eta=\eta(\omega)$, and let $u \in \mathbb{C} \backslash\{\mathbb{Q} \omega \cup \Omega\}$. Then at least two of the five numbers

$$
g_{2}, \quad g_{3}, \quad \wp(u), \quad \zeta(u)-\frac{\eta}{\omega} u, \quad \frac{\eta}{\omega}
$$

are algebraically independent. 
Chudnovsky's method was extended by K.G. Vasil'ev [225] and P. Grinspan [94], who proved that at least two of the three numbers $\pi, \Gamma(1 / 5)$, and $\Gamma(2 / 5)$ are algebraically independent. Their proof involves the Jacobian of the Fermat curve $X^{5}+Y^{5}=Z^{5}$, which contains an abelian variety of dimension 2 as a factor. See also Pellarin's papers [179, 180].

\subsection{Large Transcendence Degree}

Another important (and earlier) contribution of G.V. Chudnovsky goes back to 1974, when he worked on extending Gel'fond's method in order to prove results on large transcendence degree (see references in [50, 231]).

Chudnovsky proved that three of the numbers

$$
\alpha^{\beta}, \alpha^{\beta^{2}}, \ldots, \alpha^{\beta^{d-1}}
$$

are algebraically independent if $\alpha$ is a nonzero algebraic number, $\log \alpha$ a nonzero $\operatorname{logarithm}$ of $\alpha$, and $\beta$ an algebraic number of degree $d \geq 7$. The same year, with a much more difficult and highly technical proof, he made the first substantial progress toward a proof that there exist at least $n$ algebraically independent numbers in the set (11), provided that $d \geq 2^{n}-1$. This was a remarkable achievement since no such result providing a lower bound for the transcendence degree was known (see [235] Section 2.1). Later, thanks to the work of several mathematicians, including P. Philippon (see [182] for his trick involving the introduction of redundant variables) and Yu. V. Nesterenko [166, 167, 168], the proof was completed and the exponential lower bound for $d$ was reduced to a polynomial bound, until G. Diaz [63] obtained the best known results so far: the transcendence degree is at least $[(d+1) / 2]$.

During a short time, thanks to the work of Philippon, the elliptic results dealing with large transcendence degree where stronger than the exponential ones (see [235] p. 561).

Further results of algebraic independence related to elliptic functions are given in $[46,48,50,169,170,171,197,231]$.

In 1980, G.V. Chudnovsky [47] proved the Lindemann-Weierstrass theorem for $n=2$ and $n=3$ (small transcendence degree) by means of a clever variation of Gel'fond's method. At the same time he obtained the elliptic analogue in the CM case of the Lindemann-Weierstrass theorem for $n=2$ and $n=3$ in [46] and [47]. Also in [46] he announces further results of small transcendence degree (algebraic independence of four numbers).

This method was extended to large transcendence degree by P. Philippon [183, 184, 185] and G. Wüstholz [247, 248], who also succeeded in 1982 to prove the elliptic analogue of the Lindemann-Weierstrass theorem on the algebraic independence of $e^{\alpha_{1}}, \ldots, e^{\alpha_{n}}$ in the CM case:

Theorem 39. Let $\wp$ be a Weierstrass elliptic function with algebraic invariants $g_{2}, g_{3}$ and complex multiplication. Let $\alpha_{1}, \ldots, \alpha_{m}$ be algebraic numbers that are linearly independent over the field of endomorphisms of E. Then the numbers $\wp\left(\alpha_{1}\right), \ldots, \wp\left(\alpha_{n}\right)$ are algebraically independent. 
The same conclusion should also hold in the non-CM case; so far, only the algebraic independence of at least $n / 2$ of these numbers is known.

Further results on large transcendence degree are due to D.W. Masser and G. Wüstholz [161, 162], W.D. Brownawell [36], W.D. Brownawell and R. Tubbs [38], M. Takeuchi [216].

A survey on algebraic independence was written in 1979 by W.D. Brownawell [35]. The period prior to 1984 is covered by [235] (see also [236]), while [242] gives references for the period 1984-1997. A more recent reference is [177] Chapter 14.

\subsection{Modular Functions and Ramanujan Functions}

Ramanujan [193] introduced the following functions:

$$
\begin{aligned}
& P(q)=1-24 \sum_{n=1}^{\infty} \frac{n q^{n}}{1-q^{n}}, \quad Q(q)=1+240 \sum_{n=1}^{\infty} \frac{n^{3} q^{n}}{1-q^{n}}, \\
& R(q)=1-504 \sum_{n=1}^{\infty} \frac{n^{5} q^{n}}{1-q^{n}}
\end{aligned}
$$

They are special cases of Fourier expansions of Eisenstein series. Recall the Bernoulli numbers $B_{k}$ defined by

$$
\begin{aligned}
& \frac{z}{e^{z}-1}=1-\frac{z}{2}+\sum_{k=1}^{\infty}(-1)^{k+1} B_{k} \frac{z^{2 k}}{(2 k) !} \\
& B_{1}=1 / 6, \quad B_{2}=1 / 30, \quad B_{3}=1 / 42 .
\end{aligned}
$$

For $k \geq 1$ the normalized Eisenstein series of weight $k$ is ([116] Section 3.2, Proposition 6, [208] Section 7.4.2)

$$
E_{2 k}(q)=1+(-1)^{k} \frac{4 k}{B_{k}} \sum_{n=1}^{\infty} \frac{n^{2 k-1} q^{n}}{1-q^{n}} .
$$

The connection with (4) is

$$
E_{2 k}(q)=\frac{1}{2 \zeta(2 k)} \cdot G_{k}(\tau)
$$

for $k \geq 2$, where $q=e^{2 \pi i \tau}$ ([48] Section 3.2, Proposition 6). In particular,

$$
G_{2}(\tau)=\frac{\pi^{4}}{3^{2} \cdot 5} \cdot E_{4}(q), \quad G_{3}(\tau)=\frac{2 \pi^{6}}{3^{3} \cdot 5 \cdot 7} \cdot E_{6}(q)
$$

With Ramanujan's notation we have

$$
P(q)=E_{2}(q), \quad Q(q)=E_{4}(q), \quad R(q)=E_{6}(q) .
$$


The discriminant $\Delta$ and the modular invariant $J$ are related to these functions by Jacobi's product formula ([127] Section 18.4 and [208] Sections 7.2.3, 7.3.3, 7.4.4)

$$
\Delta=\frac{(2 \pi)^{12}}{12^{3}} \cdot\left(Q^{3}-R^{2}\right)=(2 \pi)^{12} q \prod_{n=1}^{\infty}\left(1-q^{n}\right)^{24}
$$

and

$$
J=\frac{(2 \pi)^{12} Q^{3}}{\Delta}=\frac{\left(2^{4} 3^{2} 5 G_{2}\right)^{3}}{\Delta} .
$$

Let $q$ be a complex number, $0<|q|<1$. There exists $\tau$ in the upper half-plane $\mathcal{H}$ such that $q=e^{2 \pi i \tau}$. Select any twelfth root $\omega$ of $\Delta(q)$. The invariants $g_{2}$ and $g_{3}$ of the Weierstrass $\wp$ function attached to the lattice $(\mathbb{Z}+\mathbb{Z} \tau) \omega$ satisfy $g_{2}^{3}-27 g_{3}^{2}=1$ and (see [66] Section 1.2.2 p. 163, [127], Section 4.2, Proposition 4 and Section 18.3)

$$
P(q)=3 \frac{\omega}{\pi} \cdot \frac{\eta}{\pi}, \quad Q(q)=\frac{3}{4}\left(\frac{\omega}{\pi}\right)^{4} g_{2}, \quad R(q)=\frac{27}{8}\left(\frac{\omega}{\pi}\right)^{6} g_{3} .
$$

According to formulas (5) and (6), here are a few special values (see, for instance, [4], [177] Section 3.1 and [241]).

- For $\tau=i, q=e^{-2 \pi}$,

$$
\begin{aligned}
& P\left(e^{-2 \pi}\right)=\frac{3}{\pi}, \quad Q\left(e^{-2 \pi}\right)=3\left(\frac{\omega_{1}}{\pi}\right)^{4}, \quad R\left(e^{-2 \pi}\right)=0, \quad \text { and } \\
& \Delta\left(e^{-2 \pi}\right)=2^{6} \omega_{1}^{12}
\end{aligned}
$$

with

$$
\omega_{1}=\frac{\Gamma(1 / 4)^{2}}{\sqrt{8 \pi}}=2.6220575542 \ldots
$$

- For $\tau=\varrho, q=-e^{-\pi \sqrt{3}}$,

$$
\begin{aligned}
& P\left(-e^{-\pi \sqrt{3}}\right)=\frac{2 \sqrt{3}}{\pi}, Q\left(-e^{-\pi \sqrt{3}}\right)=0, R\left(-e^{-\pi \sqrt{3}}\right)=\frac{27}{2}\left(\frac{\omega_{1}}{\pi}\right)^{6}, \\
& \Delta\left(-e^{-\pi \sqrt{3}}\right)=-2^{4} 3^{3} \omega_{1}^{12},
\end{aligned}
$$

with

$$
\omega_{1}=\frac{\Gamma(1 / 3)^{3}}{2^{4 / 3} \pi}=2.428650648 \ldots
$$

\subsection{Mahler-Manin Problem on $J(q)$}

After Schneider's theorem (Corollary 21) on the transcendence of the values of the modular function $j(\tau)$, the first results on Eisenstein series (cf. Section 5.6) go back to 1977 with D. Bertrand's work [21, 19]. See also his papers [18, 20, 23, 24], his 
work [29] with M. Laurent on values of theta functions, and Yanchenko's paper [252]. Further related results are Theorems 5 and 6 (p. 344) and Theorem 4 (p. 347) in Chudnovsky's lecture at the Helsinki ICM in 1978 [48].

The first transcendence proof using modular forms is due to a team from St Étienne (K. Barré-Sirieix, G. Diaz, F. Gramain and G. Philibert), whence the nickname théorème stéphanois for the next result; see [16] (see also [91, 92, 90] and Chapter 2 of [177]). Theorem 40 answers a conjecture of K. Mahler [139, 140] in the complex case and of Yu. V. Manin [142] in the $p$-adic case. Manin's question on the arithmetic nature of the $p$-adic number $J(q)$ is motivated by Mazur's theory, but he also asked "an obvious analogue" in the complex case; see Conjecture 43 below). We state the result only in the complex case; the paper [16] solves both cases.

Theorem 40 (K. Barré, G. Diaz, F. Gramain, G. Philibert, 1996). Let $q \in \mathbb{C}$, $0<|q|<1$. If $q$ is algebraic, then $J(q)$ is transcendental.

The solution of Manin's problem in the $p$-adic case has several consequences. It is a tool both for R. Greenberg in his study of zeros of $p$-adic $L$ functions and for H. Hida, J. Tilouine, and É. Urban in their solution of the main conjecture for the Selmer group of the symmetric square of an elliptic curve with multiplicative reduction at $p$ (references are given in [239]).

The proof of Theorem 40 involves upper bounds for the growth of the coefficients of the modular function $J(q)$. Such estimates were produced first by K. Mahler [141] Section 3. A refined estimate, due to N. Brisebarre and G. Philibert [34], for the coefficients $c_{k}(m)$ (which are nonnegative rational integers) in

$$
(q J(q))^{k}=\sum_{m=0}^{\infty} c_{k}(m) q^{m}
$$

is

$$
c_{k}(m) \leq e^{4 \sqrt{k m}} .
$$

According to a remark by D. Bertrand (Lemma 1 in [241] and Lemma 2.4 p. 17 in [177]; see also Lemma 2 in [15] and Lemma 1 in [27]), the upper bound

$$
\left|\tilde{c}_{N, k}(m)\right| \leq C^{N} m^{12 N}
$$

$(0 \leq k \leq N, N \geq 1, m \geq 1$, with an absolute constant $C$ ) for the coefficients in the Taylor development at the origin of $\Delta^{2 N} J^{k}$,

$$
\Delta(q)^{2 N} J(q)^{k}=\sum_{m=1}^{\infty} \tilde{c}_{N k}(m) q^{m},
$$

is sufficient for the proof of Theorem 40 and is an easy consequence of a theorem of Hecke ([208] Section 7.4.3, Theorem 5), together with the fact that $\Delta^{2}$ and $\Delta^{2} J$ are parabolic modular forms of weight 24 . 
One of the main tools involved in the proof of Theorem 40 is an estimate for the degrees and height of $J\left(q^{n}\right)$ in terms of $J(q)$ (which is assumed to be algebraic) and $n \geq 1$. There exists a symmetric polynomial $\Phi_{n} \in \mathbb{Z}[X, Y]$, of degree

$$
\psi(n)=n \prod_{p \mid n}\left(1+\frac{1}{p}\right)
$$

in each variable, such that $\Phi_{n}\left(J(q), J\left(q^{n}\right)\right)=0$. Again, K. Mahler was the first to investigate the coefficients of the polynomial $\Phi_{n}(X, Y)$ : in [141] he proved that its length (sum of the absolute values of the coefficients) satisfies

$$
L\left(\Phi_{n}\right) \leq e^{c n^{3 / 2}}
$$

with an absolute constant $c$. In the special case $n=2^{m}$ he had an earlier stronger result in [140], namely

$$
L\left(\Phi_{n}\right) \leq 2^{57 n} n^{36 n},
$$

and he claimed (see [140] p. 97) that if the sharper upper bound

$$
L\left(\Phi_{n}\right) \leq 2^{C n}
$$

with a positive absolute constant $C>0$ were true for $n=2^{m}$, he could prove Theorem 40. However, in 1984, P. Cohen [58] produced asymptotic estimates that show that Mahler's expectation was too optimistic:

$$
\lim _{\substack{n=2^{m} \\ m \rightarrow \infty}} \frac{1}{n \log n} \log L\left(\Phi_{n}\right)=9 .
$$

In fact she proved more precise results, without the condition $n=2^{m}$, which imply, for instance, $\log L\left(\Phi_{n}\right) \sim 6 \psi(n) \log n$ for $n \rightarrow \infty$.

Further related results are given in [67] (G. Diaz and G. Philibert) for the $j$-function and [159] (D.W. Masser) for the $\wp$-function.

The proof of [16] can be adapted to yield quantitative estimates [14, 15].

A reformulation of Theorem 40 on the transcendence of $J(q)$ is the following mixed analogue of the four exponentials conjecture (Conjecture 12):

Corollary 41. Let $\log \alpha$ be a logarithm of a nonzero algebraic number. Let $\mathbb{Z} \omega_{1}+\mathbb{Z} \omega_{2}$ be a lattice with algebraic invariants $g_{2}, g_{3}$. Then the determinant

$$
\left|\begin{array}{cc}
\omega_{1} & \log \alpha \\
\omega_{2} & 2 \pi i
\end{array}\right|
$$

does not vanish.

The four exponentials conjecture for the product of an elliptic curve by the multiplicative group is the following more general open problem: 
Conjecture 42. Let $\wp$ be a Weierstrass elliptic function with algebraic invariants $g_{2}$, $g_{3}$. Let $E$ be the corresponding elliptic curve, $u_{1}$ and $u_{2}$ two elements in $\mathcal{L}_{E}$, and $\log \alpha_{1}, \log \alpha_{2}$ two $\log$ arithms of algebraic numbers. Assume further that the two rows of the matrix

$$
M=\left(\begin{array}{l}
u_{1} \log \alpha_{1} \\
u_{2} \log \alpha_{2}
\end{array}\right)
$$

are linearly independent over $\mathbb{Q}$. Then the determinant of $M$ does not vanish.

Another special case of Conjecture 42, stronger than Corollary 41, is the next question of Yu. V. Manin, who asks in Section 4.2 of [142] to determine the nature of the invariant of the complex elliptic curve having periods 1 and a quotient $\left(\log \alpha_{1}\right) /\left(\log \alpha_{2}\right)$ of two logarithms of algebraic numbers:

Conjecture 43 (Yu.V. Manin). Let $\log \alpha_{1}$ and $\log \alpha_{2}$ be two nonzero logarithms of algebraic numbers and let $\mathbb{Z} \omega_{1}+\mathbb{Z} \omega_{2}$ be a lattice with algebraic invariants $g_{2}$ and $g_{3}$. Then

$$
\frac{\omega_{1}}{\omega_{2}} \neq \frac{\log \alpha_{1}}{\log \alpha_{2}} .
$$

In this direction let us quote some of the open problems raised by G. Diaz $[65,66]$.

Conjecture 44 (G. Diaz).

1. For any $z \in \mathbb{C}$ with $|z|=1$ and $z \neq \pm 1$, the number $e^{2 \pi i z}$ is transcendental.

2. If $q$ is an algebraic number with $0<|q|<1$ such that $J(q) \in[0,1728]$, then $q \in \mathbb{R}$.

3. The function $J$ is injective on the set of algebraic numbers $\alpha$ with $0<|\alpha|<1$.

Remark (G. Diaz). Part 3 of Conjecture 44 implies the other two and also follows from the four exponentials conjecture. It also follows from the next conjecture of D. Bertrand.

Conjecture 45 (D. Bertrand). If $\alpha_{1}$ and $\alpha_{2}$ are two multiplicatively independent algebraic numbers in the domain $\{q \in \mathbb{C} ; 0<|q|<1\}$, then the two numbers $J\left(\alpha_{1}\right)$ and $J\left(\alpha_{2}\right)$ are algebraically independent.

Conjecture 45 (see [27], where Section 5 is devoted to conjectural statements inspired by a conjecture of Oort and André) implies the special case of the four exponentials conjecture, where two of the algebraic numbers are roots of unity and the two others have modulus $\neq 1$.

\subsection{Nesterenko's Theorem}

In 1976 [18], D. Bertrand pointed out that Schneider's theorem the transcendence of $\omega / \pi$ implies the following statement: 
For any $q \in \mathbb{C}$ with $0<|q|<1$, at least one of the two numbers $Q(q), R(q)$ is transcendental.

He also proved in [18] the $p$-adic analogue by means of a new version of the Schneider-Lang criterion for meromorphic functions (he allows one essential singularity), which he applied to Jacobi-Tate elliptic functions (see also [252]). Two years later [20], he noticed that Theorem 29 yields the following:

For any $q \in \mathbb{C}$ with $0<|q|<1$, at least two of the numbers $P(q), Q(q), R(q)$ are algebraically independent.

The following result of Yu. V. Nesterenko [172, 173] (see also [175, 176, 239, 241, 32] as well as Chapters 3 and 4 of [177]) goes one step further:

Theorem 46 (Nesterenko, 1996). For any $q \in \mathbb{C}$ with $0<|q|<1$, three of the four numbers $q, P(q), Q(q), R(q)$ are algebraically independent.

Among the tools used by Nesterenko in his proof is the following result due to K. Mahler [138] (see also Chapter 1 of [177]):

The functions $P, Q, R$ are algebraically independent over $\mathbb{C}(q)$.

Also he uses the fact that they satisfy a system of differential equations for $D=$ $q d / d q$ discovered by S. Ramanujan in 1916 [193] (see also Chapters 1 and 3 of [177]):

$$
12 \frac{D P}{P}=P-\frac{Q}{P}, \quad 3 \frac{D Q}{Q}=P-\frac{R}{Q}, \quad 2 \frac{D R}{R}=P-\frac{Q^{2}}{R} .
$$

One of the main steps in his original proof $[172,173]$ is the following zero estimate:

Theorem 47 (Nesterenko's zero estimate). Let $L_{0}$ and $L$ be positive integers, $A \in$ $\mathbb{C}\left[q, X_{1}, X_{2}, X_{3}\right]$ a nonzero polynomial in four variables of degree $\leq L_{0}$ in $q$ and $\leq L$ in each of the three other variables $X_{1}, X_{2}, X_{3}$. Then the multiplicity at the origin of the analytic function $A(q, P(q), Q(q), R(q))$ is at most $2 \cdot 10^{45} L_{0} L^{3}$.

In the special case in which $J(q)$ is algebraic, P. Philippon [187] produced an alternative proof for Nesterenko's result in which this zero estimate is not used; instead of it, he used Philibert's measure of algebraic independence for $\omega / \pi$ and $\eta / \pi$ (see [181] and Section 5.2 above). However, Philibert's proof requires a zero estimate for algebraic groups.

Using (12) one deduces from Theorem 46 (see [177] Section 3.1, Corollary 1.2) the following corollary:

Corollary 48. The three numbers $\pi, e^{\pi}, \Gamma(1 / 4)$ are algebraically independent.

Using (13) one deduces (see [177] Section 1.3.1, Corollary 3.2, Remark (ii)) the following:

Corollary 49. The three numbers $\pi, e^{\pi \sqrt{3}}, \Gamma(1 / 3)$ are algebraically independent.

Consequences of Corollary 48 are the transcendence of the numbers

$$
\sigma_{\mathbb{Z}[i]}(1 / 2)=2^{5 / 4} \pi^{1 / 2} e^{\pi / 8} \Gamma(1 / 4)^{-2}
$$


and (P. Bundschuh [41])

$$
\sum_{n=0}^{\infty} \frac{1}{n^{2}+1}=\frac{1}{2}+\frac{\pi}{2} \cdot \frac{e^{\pi}+e^{-\pi}}{e^{\pi}-e^{-\pi}}
$$

D. Duverney, K. and K. Nishioka, and I. Shiokawa [68, 69, 70, 71, 72, 73] as well as D. Bertrand [27] derived from Nesterenko's theorem a number of interesting corollaries, including the following ones ([177] Chapter 3).

Corollary 50. The Rogers-Ramanujan continued fraction

$$
R R(\alpha)=1+\frac{\alpha}{1+\frac{\alpha^{2}}{1+\frac{\alpha^{3}}{1+\ddots}}}
$$

is transcendental for any algebraic $\alpha$ with $0<|\alpha|<1$.

Corollary 51. Let $\left(F_{n}\right)_{n \geq 0}$ be the Fibonacci sequence: $F_{0}=0, F_{1}=1, F_{n}=$ $F_{n-1}+F_{n-2}$. Then the number

$$
\sum_{n=1}^{\infty} \frac{1}{F_{n}^{2}}
$$

is transcendental.

Jacobi theta series ([43] Chapter V, [244] Chapter XXI and [177] Section 3.1.3) are defined by

$$
\begin{aligned}
& \theta_{2}(q)=2 q^{1 / 4} \sum_{n \geq 0} q^{n(n+1)}=2 q^{1 / 4} \prod_{n=1}^{\infty}\left(1-q^{4 n}\right)\left(1+q^{2 n}\right), \\
& \theta_{3}(q)=\sum_{n \in \mathbb{Z}} q^{n^{2}}=\prod_{n=1}^{\infty}\left(1-q^{2 n}\right)\left(1+q^{2 n-1}\right)^{2}, \\
& \theta_{4}(q)=\theta_{3}(-q)=\sum_{n \in \mathbb{Z}}(-1)^{n} q^{n^{2}}=\prod_{n=1}^{\infty}\left(1-q^{2 n}\right)\left(1-q^{2 n-1}\right)^{2}
\end{aligned}
$$

Corollary 52. Let $i, j$ and $k \in\{2,3,4\}$ with $i \neq j$. Let $q \in \mathbb{C}$ satisfy $0<|q|<1$. Then each of the two fields

$$
\mathbb{Q}\left(q, \theta_{i}(q), \theta_{j}(q), D \theta_{k}(q)\right) \quad \text { and } \mathbb{Q}\left(q, \theta_{k}(q), D \theta_{k}(q), D^{2} \theta_{k}(q)\right)
$$

has transcendence degree $\geq 3$ over $\mathbb{Q}$. 
As an example, for an algebraic number $q \in \mathbb{C}$ with $0<|q|<1$, the three numbers

$$
\sum_{n \geq 0} q^{n^{2}}, \quad \sum_{n \geq 1} n^{2} q^{n^{2}}, \quad \sum_{n \geq 1} n^{4} q^{n^{2}}
$$

are algebraically independent. In particular, the number

$$
\theta_{3}(q)=\sum_{n \in \mathbb{Z}} q^{n^{2}}
$$

is transcendental. The number $\theta_{3}(q)$ was explicitly considered by Liouville as far back as 1851 (see [174] p. 295 and [177] p. 30).

The proof by Yu. V. Nesterenko is effective and yields quantitative refinements (measures of algebraic independence): [93, 174, 187].

\subsection{Further Open Problems}

Among many open problems, we mention

- the algebraic independence of the three numbers $\pi, \Gamma(1 / 3), \Gamma(1 / 4)$.

- the algebraic independence of three numbers among $\pi, \Gamma(1 / 5), \Gamma(2 / 5), e^{\pi \sqrt{5}}$.

- the algebraic independence of the four numbers $e, \pi, e^{\pi}$ and $\Gamma(1 / 4)$.

The main conjectures in this domain are due to S. Schanuel, A. Grothendieck, Y. André [9], and C. Bertolin [17]. Chudnovsky's proof of the algebraic independence of $\pi$ and $\Gamma(1 / 4)$ involves elliptic functions; Nesterenko's proof of the algebraic independence of $\pi$ and $e^{\pi}$ requires modular functions. One may expect that higher-dimensional objects (abelian varieties, motives) may be required in order to go further. In this respect we conclude by alluding to the remarkable progress that has been achieved recently in finite characteristic (after the work by Jing Yu, G.W. Anderson and D. Thakur, L. Denis, W.D. Brownawell, J.F. Voloch, M. Papanikolas, among others).

\section{References}

1. M. ABLY - "Résultats quantitatifs d'indépendance algébrique pour les groupes algébriques," J. Number Theory 42 (1992), no. 2, pp. 194-231.

2. - , "Formes linéaires de logarithmes de points algébriques sur une courbe elliptique de type CM," Ann. Inst. Fourier (Grenoble) 50 (2000), no. 1, pp. 1-33.

3. M. ABLY \& É. GAUDRON - "Approximation diophantienne sur les courbes elliptiques à multiplication complexe," C. R. Math. Acad. Sci. Paris 337 (2003), no. 10, pp. 629-634.

4. M. ABRAmowitz \& I. A. STEGUN - Handbook of mathematical functions with formulas, graphs, and mathematical tables, A Wiley-Interscience Publication, New York: John Wiley \& Sons, Inc; Washington, D.C, 1984, Reprint of the 1972 ed.

5. M. ANDERSON - "Inhomogeneous linear forms in algebraic points of an elliptic function," in Transcendence theory: advances and applications (Proc. Conf., Univ. Cambridge, Cambridge, 1976), Academic Press, London, 1977, pp. 121-143. 
6. M. ANDERSON \& D. W. MASSER - "Lower bounds for heights on elliptic curves," Math. Z. 174 (1980), no. 1, pp. 23-34.

7. Y. ANDRÉ - G-functions and geometry, Aspects of Mathematics, E13, Friedr. Vieweg \& Sohn, Braunschweig, 1989.

8. - , "G-fonctions et transcendance," J. reine angew. Math. 476 (1996), pp. 95-125.

9. _- , Une introduction aux motifs (motifs purs, motifs mixtes, périodes), Panoramas et Synthèses, vol. 17, Société Mathématique de France, Paris, 2004.

10. A. BAKER - "On the quasi-periods of the Weierstrass $\zeta$-function," Nachr. Akad. Wiss. Göttingen Math.-Phys. Kl. II 1969 (1969), pp. 145-157.

11. - , "An estimate for the $\wp$-function at an algebraic point," Amer. J. Math. 92 (1970), pp. 619-622.

12. — , "On the periods of the Weierstrass $\wp$-function," in Symposia Mathematica, Vol. IV (INDAM, Rome, 1968/69), Academic Press, London, 1970, pp. 155-174.

13. - , Transcendental number theory, Cambridge University Press, London, 1975.

14. K. BARRÉ - "Mesures de transcendance pour l'invariant modulaire," C. R. Acad. Sci. Paris Sér. I Math. 323 (1996), no. 5, pp. 447-452.

15. - , "Mesure d'approximation simultanée de $q$ et $J(q)$," J. Number Theory 66 (1997), no. 1 , pp. 102-128.

16. K. Barré-Sirieix, G. Diaz, F. Gramain \& G. Philibert - "Une preuve de la conjecture de Mahler-Manin," Invent. Math. 124 (1996), no. 1-3, pp. 1-9.

17. C. BERTOLIN - "Périodes de 1-motifs et transcendance," J. Number Theory 97 (2002), no. 2, pp. 204-221.

18. D. BERTRAND - "Séries d'Eisenstein et transcendance," Bull. Soc. Math. France 104 (1976), no. 3, pp. 309-321.

19. - - "Transcendance de valeurs de la fonction gamma d'après G. V. Chudnovsky (Dokl. Akad. Nauk Ukrain. SSR Ser. A 1976, no. 8, 698-701)," in Séminaire Delange-PisotPoitou, 17e année (1975/76), Théorie des nombres: Fasc. 2, Exp. No. G8, Secrétariat Math., Paris, 1977, p. 5.

20. - , "Fonctions modulaires, courbes de Tate et indépendance algébrique," in Séminaire Delange-Pisot-Poitou, 19e année: 1977/78, Théorie des nombres, Fasc. 2, Secrétariat Math., Paris, 1978, p. Exp. No. 36, 11.

21. - , "Modular function and algebraic independence," in Proceedings of the Conference on p-adic Analysis (Nijmegen, 1978), Report, vol. 7806, Katholieke Univ. Nijmegen, 1978, pp. 16-23.

22. - , "Propriétés arithmétiques des dérivées de la fonction modulaire $j(\tau)$," in Séminaire de Théorie des Nombres 1977-1978, CNRS, Talence, 1978, p. Exp. No. 22, 4.

23. - , "Fonctions modulaires et indépendance algébrique. II," in Journées Arithmétiques de Luminy (Colloq. Internat. CNRS, Centre Univ. Luminy, Luminy, 1978), Astérisque, vol. 61, Soc. Math. France, Paris, 1979, pp. 29-34.

24. — , "Sur les périodes de formes modulaires," C. R. Acad. Sci. Paris Sér. A-B 288 (1979), no. 10, pp. A531-A534.

25. - " "Variétés abeliennes et formes linéaires d'intégrales elliptiques," in Théorie des nombres, Sémin. Delange-Pisot-Poitou, Paris 1979-80, Prog. Math. 12, 15-27, 1981.

26. — , "Endomorphismes de groupes algébriques; applications arithmétiques," in Diophantine approximations and transcendental numbers (Luminy, 1982), Progr. Math., vol. 31, Birkhäuser Boston, Boston, MA, 1983, pp. 1-45.

27. - , "Theta functions and transcendence," Ramanujan J. 1 (1997), no. 4, pp. 339-350, International Symposium on Number Theory (Madras, 1996).

28. D. BERTRAND \& Y. Z. FLICKER - "Linear forms on abelian varieties over local fields," Acta Arith. 38 (1980/81), no. 1, pp. 47-61. 
29. D. Bertrand \& M. LAURENT - "Propriétés de transcendance de nombres liés aux fonctions thêta," C. R. Acad. Sci. Paris Sér. I Math. 292 (1981), no. 16, pp. 747-749.

30. D. Bertrand \& D. MASSER - "Formes linéaires d'intégrales abéliennes," C. R. Acad. Sci. Paris Sér. A-B 290 (1980), no. 16, pp. A725-A727.

31. - , "Linear forms in elliptic integrals," Invent. Math. 58 (1980), no. 3, pp. 283-288.

32. V. Bosser - "Indépendance algébrique de valeurs de séries d'Eisenstein (théorème de Nesterenko)," in Formes modulaires et transcendance, Sémin. Congr., vol. 12, Soc. Math. France, Paris, 2005, pp. 119-178.

33. J.-B. Bost - "Périodes et isogénies des variétés abéliennes sur les corps de nombres (d'après D. Masser et G. Wüstholz)," Astérisque 237 (1996), no. 4, pp. 115-161, Séminaire Bourbaki, Vol. 1994/95, Exp. No. 795.

34. N. BrisebarRe \& G. Philibert - "Effective lower and upper bounds for the Fourier coefficients of powers of the modular invariant $j$," Journal of the Ramanujan Mathematical Society 20 (2005), no. 4, pp. 255-282.

35. W. D. BROWNAWELL - "On the development of Gel'fond's method," in Number theory, Carbondale 1979 (Proc. Southern Illinois Conf., Southern Illinois Univ., Carbondale, Ill., 1979), Lecture Notes in Math., vol. 751, Springer, Berlin, 1979, pp. 18-44.

36. - , "Large transcendence degree revisited. I. Exponential and non-CM cases," in Diophantine approximation and transcendence theory (Bonn, 1985), Lecture Notes in Math., vol. 1290, Springer, Berlin, 1987, pp. 149-173.

37. W. D. BRownAWELl \& K. K. KuBOtA - "The algebraic independence of Weierstrass functions and some related numbers," Acta Arith. 33 (1977), no. 2, pp. 111-149.

38. W. D. BROWNAWELL \& R. TUBBS - "Large transcendence degree revisited. II. The CM case," in Diophantine approximation and transcendence theory (Bonn, 1985), Lecture Notes in Math., vol. 1290, Springer, Berlin, 1987, pp. 175-188.

39. S. BRUILTET - "D'une mesure d'approximation simultanée à une mesure d'irrationalité: le cas de $\Gamma(1 / 4)$ et $\Gamma(1 / 3)$," Acta Arith. 104 (2002), no. 3, pp. 243-281.

40. P. BundsCHUH - "Ein Approximationsmass für transzendente Lösungen gewisser transzendenter Gleichungen," J. reine angew. Math. 251 (1971), pp. 32-53.

41. — , "Zwei Bemerkungen über transzendente Zahlen," Monatsh. Math. 88 (1979), no. 4, pp. 293-304.

42. E. B. BURGER \& R. TUbBS - Making transcendence transparent, Springer-Verlag, New York, 2004, An intuitive approach to classical transcendental number theory.

43. K. Chandrasekharan - Elliptic functions, Grundlehren der Mathematischen Wissenschaften, vol. 281, Springer-Verlag, Berlin, 1985.

44. S. Chowla \& A. Selberg - "On Epstein's zeta-function," J. reine angew. Math. 227 (1967), pp. 86-110.

45. G. V. CHUDNOVSKY - "Algebraic independence of constants connected with the exponential and the elliptic functions," Dokl. Akad. Nauk Ukrain. SSR Ser. A 8 (1976), pp. 698-701, 767.

46. - , "Indépendance algébrique des valeurs d'une fonction elliptique en des points algébriques. Formulation des résultats," C. R. Acad. Sci. Paris Sér. A-B 288 (1979), no. 8, pp. A439-A440.

47. - "Algebraic independence of the values of elliptic function at algebraic points," Invent. Math. 61 (1980), no. 3, pp. 267-290, Elliptic analogue of the LindemannWeierstrass theorem.

48. - , "Algebraic independence of values of exponential and elliptic functions," in Proceedings of the International Congress of Mathematicians (Helsinki, 1978) (Helsinki), Acad. Sci. Fennica, 1980, pp. 339-350. 
49. - - "Indépendance algébrique dans la méthode de Gelfond-Schneider," C. R. Acad. Sci., Paris, Sér. A 291 (1980), pp. 365-368, see Zbl 0456.10016.

50. - , Contributions to the theory of transcendental numbers, Mathematical Surveys and Monographs, vol. 19, American Mathematical Society, Providence, RI, 1984.

51. J. COATES - "An application of the division theory of elliptic functions to Diophantine approximation," Invent. Math. 11 (1970), pp. 167-182.

52. - , "An application of the Thue-Siegel-Roth theorem to elliptic functions," Proc. Cambridge Philos. Soc. 69 (1971), pp. 157-161.

53. - , "Linear forms in the periods of the exponential and elliptic functions," Invent. Math. 12 (1971), pp. 290-299.

54. - , "The transcendence of linear forms in $\omega_{1}, \omega_{2}, \eta_{1}, \eta_{2}, 2 \pi i$," Amer. J. Math. 93 (1971), pp. 385-397.

55. - , "Linear relations between $2 \pi i$ and the periods of two elliptic curves," in Diophantine approximation and its applications (Proc. Conf., Washington, D.C., 1972), Academic Press, New York, 1973, pp. 77-99.

56. J. COATES \& S. LANG - "Diophantine approximation on Abelian varieties with complex multiplication," Invent. Math. 34 (1976), no. 2, pp. 129-133 (= [130] pp. 236-240).

57. H. CoHEN - "Elliptic curves," in From Number Theory to Physics (Les Houches, 1989), Springer, Berlin, 1992, pp. 212-237.

58. P. B. COHEN - "On the coefficients of the transformation polynomials for the elliptic modular function," Math. Proc. Cambridge Philos. Soc. 95 (1984), no. 3, pp. 389-402.

59. - , "Perspectives de l'approximation diophantienne et de la transcendance," Ramanujan J. 7 (2003), no. 1-3, pp. 367-384, Rankin memorial issues.

60. S. DAVID - "Minorations de formes linéaires de logarithmes elliptiques," Mém. Soc. Math. France (N.S.) 62 (1995), pp. 1-143.

61. - , "On the height of subvarieties of group varieties," The Ramanujan Journal of Math., to appear, 2005.

62. S. DAVID \& N. HIRATA-KoHNO - "Recent progress on linear forms in elliptic logarithms," in A panorama of number theory or the view from Baker's garden (Zürich, 1999), Cambridge Univ. Press, Cambridge, 2002, pp. 26-37.

63. G. DiAZ - "Grands degrés de transcendance pour des familles d'exponentielles," J. Number Theory 31 (1989), no. 1, pp. 1-23.

64. — , "Minorations de combinaisons linéaires non homogènes pour un logarithme elliptique," C. R. Acad. Sci. Paris Sér. I Math. 318 (1994), no. 10, pp. 879-883.

65. — , "La conjecture des quatre exponentielles et les conjectures de D. Bertrand sur la fonction modulaire," J. Théor. Nombres Bordeaux 9 (1997), no. 1, pp. 229-245.

66. - - "Transcendance et indépendance algébrique: liens entre les points de vue elliptique et modulaire," Ramanujan J. 4 (2000), no. 2, pp. 157-199.

67. G. Diaz \& G. Philibert - "Growth properties of the modular function $j$," J. Math. Anal. Appl. 139 (1989), no. 2, pp. 382-389.

68. D. Duverney, K. Nishioka, K. Nishioka \& I. Shiokawa - "Transcendence of Jacobi's theta series," Proc. Japan Acad. Ser. A Math. Sci. 72 (1996), no. 9, pp. 202-203.

69. - , "Transcendence of Rogers-Ramanujan continued fraction and reciprocal sums of Fibonacci numbers," Proc. Japan Acad. Ser. A Math. Sci. 73 (1997), no. 7, pp. 140-142.

70. - , "Transcendence of Jacobi's theta series and related results," in Number theory (Eger, 1996), W. de Gruyter, Berlin, 1998, pp. 157-168.

71. - , "Transcendence of Rogers-Ramanujan continued fraction and reciprocal sums of Fibonacci numbers," Sūrikaisekikenkyūsho Kōkyūroku 1060 (1998), pp. 91-100, Number theory and its applications (Japanese) (Kyoto, 1997). 
72. D. DuVERnEy \& I. Shiokawa - "On some arithmetical properties of RogersRamanujan continued fraction," in Colloque Franco-Japonais: Théorie des Nombres Transcendants (Tokyo, 1998), Sem. Math. Sci., vol. 27, Keio Univ., Yokohama, 1999, pp. $91-100$.

73. - - "On some arithmetical properties of Rogers-Ramanujan continued fraction," Osaka J. Math. 37 (2000), no. 3, pp. 759-771.

74. A. FAisAnt \& G. PhiliberT - "Mesure d'approximation simultanée pour la fonction modulaire $j$ et résultats connexes," in Séminaire de théorie des nombres, Paris 1984-85, Progr. Math., vol. 63, Birkhäuser Boston, Boston, MA, 1986, pp. 67-78.

75. - , "Quelques résultats de transcendance liés à l'invariant modulaire $j$, , J. Number Theory 25 (1987), no. 2, pp. 184-200.

76. N. I. FEL'DMAN - "On the measure of transcendency of the logarithms of algebraic numbers and elliptic constants," Uspehi Matem. Nauk (N.S.) 4 (1949), no. 1(29), p. 190.

77. — " "The approximation of certain transcendental numbers. II. The approximation of certain numbers connected with the Weierstrass function $\wp(z)$," Izvestiya Akad. Nauk SSSR. Ser. Mat. 15 (1951), pp. 153-176.

78. - , "Joint approximations of the periods of an elliptic function by algebraic numbers," Izv. Akad. Nauk SSSR Ser. Mat. 22 (1958), pp. 563-576.

79. - , "An elliptic analog of an inequality of A. O. Gel'fond," Trudy Moskov. Mat. Obšč. 18 (1968), pp. 65-76.

80. — , "The periods of elliptic functions," Acta Arith. 24 (1973/74), pp. 477-489, Collection of articles dedicated to Carl Ludwig Siegel on the occasion of his seventyfifth birthday, V.

81. - , "The algebraic independence of certain numbers," Vestnik Moskov. Univ. Ser. I Mat. Mekh. (1980), no. 4, pp. 46-50, 100.

82. - , "Algebraic independence of some numbers. II," Ann. Univ. Sci. Budapest. Eötvös Sect. Math. 25 (1982), pp. 109-123.

83. N. I. Fel'dman \& Y. V. Nesterenko - "Transcendental numbers," in Number theory, IV, Encyclopaedia Math. Sci., vol. 44, Springer, Berlin, 1998, pp. 1-345.

84. Y. Z. FLICKER - "Linear forms on arithmetic abelian varieties: ineffective bounds," Mém. Soc. Math. France (N.S.) 2 (1980/81), pp. 41-47, Abelian functions and transcendental numbers (Colloq., École Polytech., Palaiseau, 1979).

85. R. FRANKLIN - "The transcendence of linear forms in $\omega_{1}, \omega_{2}, \eta_{1}, \eta_{2}, 2 \pi i, \log \gamma$," Acta Arith. 26 (1974/75), pp. 197-206.

86. É. GAUDRON - "Étude du cas rationnel de la théorie des formes linéaires de logarithmes," arXiv: math.NT/0410082, 2004.

Au: Current status?

87. - , "Formes linéaires de logarithmes effectives sur les variétés abéliennes," submitted, 2004.

88. - - "Mesures d'indépendance linéaire de logarithmes dans un groupe algébrique commutatif," Invent. Math. 162 (2005), no. 1, pp. 137-188.

89. A. O. GEL'FOND - Transcendental and algebraic numbers, Translated from the first Russian edition by Leo F. Boron, Dover Publications Inc., New York, 1960.

90. P. GRAFTIEAUX - "Théorème stéphanois et méthode des pentes," in Formes modulaires et transcendance, Sémin. Congr., vol. 12, Soc. Math. France, Paris, 2005, pp. 179-213.

91. F. GRAmAIN - "Quelques résultats d'indépendance algébrique," in Proceedings of the International Congress of Mathematicians, Vol. II (Berlin), 1998, pp. 173-182.

92. — , "Transcendance et fonctions modulaires," J. Théor. Nombres Bordeaux 11 (1999), no. 1, pp. 73-90, Les XXèmes Journées Arithmétiques (Limoges, 1997).

93. P. GRINSPAN - "A measure of simultaneous approximation for quasi-modular functions," Ramanujan J. 5 (2001), no. 1, pp. 21-45. 
94. - , "Measures of simultaneous approximation for quasi-periods of abelian varieties," J. Number Theory 94 (2002), no. 1, pp. 136-176.

95. B. H. GRoss - "On the periods of abelian integrals and a formula of Chowla and Selberg," Invent. Math. 45 (1978), no. 2, pp. 193-211, With an appendix by David E. Rohrlich.

96. — , "On an identity of Chowla and Selberg," J. Number Theory 11 (1979), no. 3 S. Chowla Anniversary Issue, pp. 344-348.

97. T. HARASE - "The transcendence of $e^{\alpha \omega+\beta(2 \pi i)}, " J$. Fac. Sci. Univ. Tokyo Sect. IA Math. 21 (1974), pp. 279-285.

98. - , "On the linear form of transcendental numbers; $\alpha_{1} \omega+\alpha_{3} \log \alpha_{4}$," J. Fac. Sci. Univ. Tokyo Sect. IA Math. 23 (1976), no. 3, pp. 435-452.

99. M. HINDRY \& J. H. Silverman - Diophantine geometry, Graduate Texts in Mathematics, vol. 201, Springer-Verlag, New York, 2000, An introduction.

100. N. Hirata-Kohno - "Formes linéaires d'intégrales elliptiques," in Séminaire de Théorie des Nombres, Paris 1988-1989, Progr. Math., vol. 91, Birkhäuser Boston, Boston, MA, 1990, pp. 117-140.

101. - , "Mesures de transcendance pour les quotients de périodes d'intégrales elliptiques," Acta Arith. 56 (1990), no. 2, pp. 111-133.

102. - , "Formes linéaires de logarithmes de points algébriques sur les groupes algébriques," Invent. Math. 104 (1991), no. 2, pp. 401-433.

103. - , "Nouvelles mesures de transcendance liées aux groupes algébriques commutatifs," in Approximations diophantiennes et nombres transcendants (Luminy, 1990), W. de Gruyter, Berlin, 1992, pp. 165-172.

104. — , "Approximations simultanées sur les groupes algébriques commutatifs," Compositio Math. 86 (1993), no. 1, pp. 69-96.

105. E. M. JABBOURI - "Mesures d'indépendance algébrique de valeurs de fonctions elliptiques et abéliennes," C. R. Acad. Sci. Paris Sér. I Math. 303 (1986), no. 9, pp. 375-378.

106. Y. M. KHOLYAVKA - "Simultaneous approximations of the invariants of an elliptic function by algebraic numbers," in Diophantine approximations, Part II (Russian), Moskov. Gos. Univ., Moscow, 1986, pp. 114-121.

107. - , "Approximation of numbers that are connected with elliptic functions," Mat. Zametki 47 (1990), no. 6, pp. 110-118, 160.

108. — , "Approximation of the invariants of an elliptic function," Ukrain. Mat. Zh. 42 (1990), no. 5, pp. 681-685.

109. - , "On the approximation of some numbers related to $\wp(z), "$ Vìsnik $L^{\prime} v \bar{v} v$. Unīv. Ser. Mekh. Mat. 34 (1990), pp. 88-89.

110. - , "On the approximation of numbers connected with Weierstrass elliptic functions," Sibirsk. Mat. Zh. 32 (1991), no. 1, pp. 212-216, 224.

111. - , "Simultaneous approximation of numbers connected with elliptic functions," Izv. Vyssh. Uchebn. Zaved. Mat. 3 (1991), pp. 70-73.

112. — , "Zeros of polynomials of Jacobi elliptic functions," Ukraïn. Mat. Zh. 44 (1992), no. 11, p. 1624.

113. - , "On the approximation of certain numbers associated with Jacobi elliptic functions," in Mathematical investigations (Ukrainian), Pr. L'vīv. Mat. Tov., vol. 2, L'vīv. Mat. Tov., L'viv, 1993, pp. 10-13, 106.

114. - , "On the approximation of numbers connected with $\wp(z), " V \bar{s} s n i k L^{\prime} v \bar{v} v$. Unīv. Ser. Mekh. Mat. 38 (1993), p. 64.

115. N. Koblitz - "Gamma function identities and elliptic differentials on Fermat curves," Duke Math. J. 45 (1978), no. 1, pp. 87-99. 
116. - , Introduction to elliptic curves and modular forms, second ed., Graduate Texts in Mathematics, vol. 97, Springer-Verlag, New York, 1993.

117. N. Koblitz \& D. Rohrlich - "Simple factors in the Jacobian of a Fermat curve," Canad. J. Math. 30 (1978), no. 6, pp. 1183-1205.

118. M. KontSEVICH \& D. ZAGIER - "Periods," in Mathematics unlimited-2001 and beyond, Springer, Berlin, 2001, pp. 771-808.

119. S. LANG - "Diophantine approximations on toruses," Amer. J. Math. 86 (1964), pp. 521-533 (= [129] pp. 313-325).

120. - - Introduction to transcendental numbers, Addison-Wesley Publishing Co., Reading, Mass.-London-Don Mills, Ont., 1966 (= [129] pp. 396-506).

121. - , "Transcendental numbers and Diophantine approximations," Bull. Amer. Math. Soc. 77 (1971), pp. 635-677 (= [130] pp. 1-43).

122. _ , "Higher-dimensional Diophantine problems," Bull. Amer. Math. Soc. 80 (1974), pp. 779-787 (= [130] pp. 102-110).

123. - , "Diophantine approximation on abelian varieties with complex multiplication," Advances in Math. 17 (1975), no. 3, pp. 281-336 (= [130] pp. 113-168).

124. - , Elliptic curves: Diophantine analysis, Grundlehren der Mathematischen Wissenschaften, vol. 231, Springer-Verlag, Berlin, 1978.

125. - , "Relations de distributions et exemples classiques," in Séminaire Delange-PisotPoitou, 19e année: 1977/78, Théorie des nombres, Fasc. 2, Secrétariat Math., Paris, 1978, p. Exp. No. 40, 6 (= [131] pp. 59-65).

126. - , "Conjectured Diophantine estimates on elliptic curves," in Arithmetic and geometry, Vol. I, Progr. Math., vol. 35, Birkhäuser Boston, Boston, MA, 1983, pp. 155-171 (= [131] pp. 212-228).

127. — , Elliptic functions, second ed., Graduate Texts in Mathematics, vol. 112, SpringerVerlag, New York, 1987, With an appendix by J. Tate.

128. - , Cyclotomic fields I and II, second ed., Graduate Texts in Mathematics, vol. 121, Springer-Verlag, New York, 1990, With an appendix by Karl Rubin.

129. —, Collected papers. Vol. I, Springer-Verlag, New York, 2000, 1952-1970.

130. —, Collected papers. Vol. II, Springer-Verlag, New York, 2000, 1971-1977.

131. —, Collected papers. Vol. III, Springer-Verlag, New York, 2000, 1978-1990.

132. M. LAurent - "Transcendance de périodes d'intégrales elliptiques," C. R. Acad. Sci. Paris Sér. A-B 288 (1979), no. 15, pp. 699-701.

133. - ,Approximation diophantienne de valeurs de la fonction Beta aux points rationnels," Ann. Fac. Sci. Toulouse Math. (5) 2 (1980), no. 1, pp. 53-65.

134. - - "Indépendance linéaire de valeurs de fonctions doublement quasi-périodiques," C. R. Acad. Sci. Paris Sér. A-B 290 (1980), no. 9, pp. A397-A399.

135. - , "Transcendance de périodes d'intégrales elliptiques," J. reine angew. Math. 316 (1980), pp. 122-139.

136. - - "Transcendance de périodes d'intégrales elliptiques," in Séminaire Delange-PisotPoitou, 20e année: 1978/1979. Théorie des nombres, Fasc. 1, Secrétariat Math., Paris, 1980, p. Exp. No. 13, 4.

137. — , "Transcendance de périodes d'intégrales elliptiques. II," J. reine angew. Math. 333 (1982), pp. 144-161.

138. K. MAHLER - "On algebraic differential equations satisfied by automorphic functions," J. Austral. Math. Soc. 10 (1969), pp. 445-450.

139. - , "Remarks on a paper by W. Schwarz," J. Number Theory 1 (1969), pp. 512-521.

140. - , "On the coefficients of the $2^{n}$-th transformation polynomial for $j(\omega)$," Acta Arith. 21 (1972), pp. 89-97. 
141. — , "On the coefficients of transformation polynomials for the modular function," Bull. Austral. Math. Soc. 10 (1974), pp. 197-218.

142. Y. MANIN - "Cyclotomic fields and modular curves," Uspekhi Mat. Nauk 26 (1971), no. 6, pp. 7-71, Engl. Transl. Russ. Math. Surv. 26 (1971), no. 6, pp. 7-78.

143. D. W. MASSER - "On the periods of the exponential and elliptic functions," Proc. Cambridge Philos. Soc. 73 (1973), pp. 339-350.

144. - , Elliptic functions and transcendence, Springer-Verlag, Berlin, 1975, Lecture Notes in Mathematics, Vol. 437.

145. - , "Linear forms in algebraic points of Abelian functions. I," Math. Proc. Cambridge Philos. Soc. 77 (1975), pp. 499-513.

146. - , "On the periods of Abelian functions in two variables," Mathematika 22 (1975), no. 2, pp. 97-107.

147. —, "Sur les points algébriques d'une variété abélienne," C. R. Acad. Sci. Paris Sér. A-B 280 (1975), pp. A11-A12.

148. - , "Transcendence and abelian functions," in Journées Arithmétiques de Bordeaux (Conf., Univ. Bordeaux, Bordeaux, 1974), Soc. Math. France, Paris, 1975, pp. 177-182. Astérisque, Nos. 24-25.

149. - , "Linear forms in algebraic points of Abelian functions. II," Math. Proc. Cambridge Philos. Soc. 79 (1976), no. 1, pp. 55-70.

150. — , "Linear forms in algebraic points of Abelian functions. III," Proc. London Math. Soc. (3) 33 (1976), no. 3, pp. 549-564.

151. - , "A note on a paper of Richard Franklin: [85]," Acta Arith. 31 (1976), no. 2, pp. $143-152$.

152. - , "Division fields of elliptic functions," Bull. London Math. Soc. 9 (1977), no. 1, pp. 49-53.

153. - , "A note on Abelian functions," in Transcendence theory: advances and applications (Proc. Conf., Univ. Cambridge, Cambridge, 1976), Academic Press, London, 1977, pp. $145-147$.

154. - - "Some vector spaces associated with two elliptic functions," in Transcendence theory: advances and applications (Proc. Conf., Univ. Cambridge, Cambridge, 1976), Academic Press, London, 1977, pp. 101-119.

155. - , "The transcendence of certain quasi-periods associated with Abelian functions in two variables," Compositio Math. 35 (1977), no. 3, pp. 239-258.

156. - , "The transcendence of definite integrals of algebraic functions," in Journées Arithmétiques de Caen (Univ. Caen, 1976), Soc. Math. France, Paris, 1977, pp. 231-238. Astérisque No. 41-42.

157. - , "Diophantine approximation and lattices with complex multiplication," Invent. Math. 45 (1978), no. 1, pp. 61-82.

158. - , "Heights, transcendence, and linear independence on commutative group varieties," in Diophantine approximation (Cetraro, 2000), Lecture Notes in Math., vol. 1819, Springer, Berlin, 2003, pp. 1-51.

159. - , "Sharp estimates for Weierstrass elliptic functions," J. Anal. Math. 90 (2003), pp. 257-302.

160. D. W. MASSER \& G. WÜSTHOLZ - "Algebraic independence properties of values of elliptic functions," in Journées arithmétiques, Exeter 1980, Lond. Math. Soc. Lect. Note Ser. 56, 360-363, 1982.

161. - , "Fields of large transcendence degree generated by values of elliptic functions," Invent. Math. 72 (1983), no. 3, pp. 407-464.

162. - , "Algebraic independence of values of elliptic functions," Math. Ann. 276 (1986), no. 1, pp. 1-17. 
163. — , "Estimating isogenies on elliptic curves," Invent. Math. 100 (1990), no. 1, pp. 1-24.

164. - , "Galois properties of division fields of elliptic curves," Bull. London Math. Soc. 25 (1993), no. 3, pp. 247-254.

165. N. D. NAGAEV - "Approximation of the transcendental quotient of two algebraic points of the function $\wp(z)$ with complex multiplication," Mat. Zametki 20 (1976), no. 1, pp. 47-60.

166. J. V. NeStERENKO - "On the algebraical independence of algebraic numbers to algebraic powers," in Diophantine approximations and transcendental numbers (Luminy, 1982), Progr. Math., vol. 31, Birkhäuser Boston, Mass., 1983, pp. 199-220.

167. Y. V. NESTERENKO - "Algebraic independence of algebraic powers of algebraic numbers," Mat. Sb. (N.S.) 123(165) (1984), no. 4, pp. 435-459.

168. - , "Degrees of transcendence of some fields that are generated by values of an exponential function," Mat. Zametki 46 (1989), no. 3, pp. 40-49, 127.

169. Y. V. NESTERENKO - "Measure of algebraic independence of values of an elliptic function at algebraic points," Uspekhi Mat. Nauk 40 (1985), no. 4(244), pp. 221-222.

170. - , "On a measure of algebraic independence of the values of elliptic functions," in Approximations diophantiennes et nombres transcendants (Luminy, 1990), W. de Gruyter, Berlin, 1992, pp. 239-248.

171. - , "On the measure of algebraic independence of values of an elliptic function," Izv. Ross. Akad. Nauk Ser. Mat. 59 (1995), no. 4, pp. 155-178.

172. - , "Modular functions and transcendence problems," C. R. Acad. Sci. Paris Sér. I Math. 322 (1996), no. 10, pp. 909-914.

173. - , "Modular functions and transcendence questions," Mat. Sb. 187 (1996), no. 9, pp. 65-96.

174. - - "On the measure of algebraic independence of values of Ramanujan functions," $T r$. Mat. Inst. Steklova 218 (1997), no. Anal. Teor. Chisel i Prilozh., pp. 299-334.

175. - , "Algebraic independence of $\pi$ and $e^{\pi}$," in Number theory and its applications (Ankara, 1996), Lecture Notes in Pure and Appl. Math., vol. 204, Dekker, New York, 1999, pp. 121-149.

176. - , "On the algebraic independence of values of Ramanujan functions," Vestnik Moskov. Univ. Ser. I Mat. Mekh. 2 (2001), pp. 6-10, 70.

177. Y. V. NESTERENKO \& P. PHILIPPON - Introduction to algebraic independence theory, Lecture Notes in Mathematics, vol. 1752, Springer-Verlag, Berlin, 2001, With contributions from F. Amoroso, D. Bertrand, W. D. Brownawell, G. Diaz, M. Laurent, Yuri V. Nesterenko, K. Nishioka, Patrice Philippon, G. Rémond, D. Roy and M. Waldschmidt, edited by Nesterenko and Philippon.

178. F. PELLARIN - "The isogeny theorem and the irreducibility theorem for elliptic curves: a survey," Rend. Sem. Mat. Univ. Politec. Torino 53 (1995), no. 4, pp. 389-404, Number theory, II (Rome, 1995).

179. - , "The Ramanujan property and some of its connections with Diophantine geometry," Riv. Mat. Univ. Parma (7) 3* (2004), pp. 275-288.

180. — , "Introduction aux formes modulaires de Hilbert et à leurs propriétés différentielles," in Formes modulaires et transcendance, Sémin. Congr., vol. 12, Soc. Math. France, Paris, 2005, pp. 215-269.

181. G. PhILIBERT - "Une mesure d'indépendance algébrique," Ann. Inst. Fourier (Grenoble) 38 (1988), no. 3, pp. 85-103.

182. P. PHILIPPON - "Indépendance algébrique de valeurs de fonctions elliptiques p-adiques," in Proceedings of the Queen's Number Theory Conference, 1979 (Kingston, Ont., 1979) (Kingston, Ont.), Queen's Papers in Pure and Appl. Math., vol. 54, Queen's Univ., 1980, pp. 223-235. 
183. —, "Indépendance algébrique et variétés abéliennes," C. R. Acad. Sci. Paris Sér. I Math. 294 (1982), no. 7, pp. 257-259.

184. — , "Variétés abéliennes et indépendance algébrique. I," Invent. Math. 70 (1982/83), no. 3, pp. 289-318.

185. - , "Variétés abéliennes et indépendance algébrique. II. Un analogue abélien du théorème de Lindemann-Weierstrass," Invent. Math. 72 (1983), no. 3, pp. 389-405.

186. — , "Une approche méthodique pour la transcendance et l'indépendance algébrique de valeurs de fonctions analytiques," J. Number Theory 64 (1997), no. 2, pp. 291-338.

187. - , "Indépendance algébrique et $K$-fonctions," J. reine angew. Math. 497 (1998), pp. 1-15.

188. P. PHILIPPON \& M. WALDSCHMIDT - "Formes linéaires de logarithmes sur les groupes algébriques commutatifs," Illinois J. Math. 32 (1988), no. 2, pp. 281-314.

189. - , "Formes linéaires de logarithmes elliptiques et mesures de transcendance," in Théorie des nombres (Québec, PQ, 1987), W. de Gruyter, Berlin, 1989, pp. 798-805.

190. J. Popken \& K. MAHLER - "Ein neues Prinzip Transzendenzbeweise," Proc. Akad. Wet. Amsterdam 38 (1935), pp. 864-871.

191. K. RAMACHANDRA - "Contributions to the theory of transcendental numbers. I, II," Acta Arith. 14 (1967/68), 65-72; ibid. 14 (1967/1968), pp. 73-88.

192. — , Lectures on transcendental numbers, The Ramanujan Institute Lecture Notes, vol. 1, The Ramanujan Institute, Madras, 1969.

193. S. Ramanujan - "On certain arithmetical functions," Trans. Camb. Phil. Soc. 22 (1916), pp. 159-184, Collected Papers of Srinivasa Ramanujan, Chelsea Publ., N.Y. $1927, N^{\circ} 18,136-162$.

194. G. RÉMOND \& F. URFELS - "Approximation diophantienne de logarithmes elliptiques p-adiques," J. Number Theory 57 (1996), no. 1, pp. 133-169.

195. É. REYSSAT - "Mesures de transcendance de nombres liés aux fonctions elliptiques," in Séminaire Delange-Pisot-Poitou, 18e année: 1976/77, Théorie des nombres, Fasc. 2, Secrétariat Math., Paris, 1977, p. Exp. No. G22, 3.

196. — , "Mesures de transcendance de nombres liés aux fonctions exponentielles et elliptiques," C. R. Acad. Sci. Paris Sér. A-B 285 (1977), no. 16, pp. A977-A980.

197. — , "Travaux récents de G. V. Chudnovsky," in Séminaire Delange-Pisot-Poitou, 18e année: 1976/77, Théorie des nombres, Fasc. 2, Secrétariat Math., Paris, 1977, p. Exp. No. $29,7$.

198. — , "Approximation algébrique de nombres liés aux fonctions elliptiques et exponentielle," Bull. Soc. Math. France 108 (1980), no. 1, pp. 47-79.

199. — , "Approximation de nombres liés à la fonction sigma de Weierstrass," Ann. Fac. Sci. Toulouse Math. (5) 2 (1980), no. 1, pp. 79-91.

200. - , "Fonctions de Weierstrass et indépendance algébrique," C. R. Acad. Sci. Paris Sér. $A-B 290$ (1980), no. 10, pp. A439-A441.

201. - , "Propriétés d'indépendance algébrique de nombres liés aux fonctions de Weierstrass," Acta Arith. 41 (1982), no. 3, pp. 291-310.

202. N. SARADHA - "Transcendence measure for $\eta / \omega, "$ Acta Arith 92 (2000), no. 1, pp. 11-25.

203. T. SCHNEIDER - "Transzendenzuntersuchungen periodischer Funktionen. II. Transzendenzeigenschaften elliptischer Funktionen,” J. reine angew. Math. 172 (1934), pp. 70-74.

204. - , "Arithmetische Untersuchungen elliptischer Integrale," Math. Ann. 113 (1936), pp. 1-13.

205. - , "Zur Theorie der Abelschen Funktionen und Integrale," J. reine angew. Math. 183 (1941), pp. 110-128. 
206. — , "Ein Satz über ganzwertige Funktionen als Prinzip für Transzendenzbeweise," Math. Ann. 121 (1949), pp. 141-140.

207. - - Einführung in die transzendenten Zahlen, Springer-Verlag, Berlin, 1957.

208. J.-P. SERRE - Cours d'arithmétique, Collection SUP: "Le Mathématicien," vol. 2, Presses Universitaires de France, Paris, 1970, reprinted 1977. Engl. transl.: A course in arithmetic, Graduate Texts in Mathematics, Vol. 7. Springer-Verlag, New York, 1978.

209. S. O. SheStAKOV - "On the measure of algebraic independence of some numbers," Vestnik Moskov. Univ. Ser. I Mat. Mekh. 2 (1992), pp. 8-12, 111.

210. C. L. Siegel - “Über die Perioden elliptischer Funktionen,” J. f. M. 167 (1932), pp. 62-69.

211. - , Transcendental Numbers, Annals of Mathematics Studies, no. 16, Princeton University Press, Princeton, N. J., 1949.

212. J. H. SILVERMAN - The arithmetic of elliptic curves, Graduate Texts in Mathematics, vol. 106, Springer-Verlag, New York, 1986.

213. - , Advanced topics in the arithmetic of elliptic curves, Graduate Texts in Mathematics, vol. 151, Springer-Verlag, New York, 1994.

214. A. A. ŚMELEV - "The algebraic independence of the values of the exponential and an elliptic function," Mat. Zametki 20 (1976), no. 2, pp. 195-202.

215. - , "Algebraic independence of several numbers connected with exponential and elliptic functions," Ukrain. Mat. Zh. 33 (1981), no. 2, pp. 277-282.

216. M. TAKEUCHI - "Quantitative results of algebraic independence and Baker's method," Acta Arith. 119 (2005), no. 3, pp. 211-241.

217. R. TiJdEMAN - "On the algebraic independence of certain numbers," Nederl. Akad. Wetensch. Proc. Ser. A 74=Indag. Math. 33 (1971), pp. 146-162.

218. M. TOYODA \& T. YASUDA - "On the algebraic independence of certain numbers connected with the exponential and the elliptic functions," Tokyo J. Math. 9 (1986), no. 1 , pp. $29-40$.

219. R. TUBBS - "A transcendence measure for some special values of elliptic functions," Proc. Amer. Math. Soc. 88 (1983), no. 2, pp. 189-196.

220. - , "On the measure of algebraic independence of certain values of elliptic functions," J. Number Theory 23 (1986), no. 1, pp. 60-79.

221. - , "Algebraic groups and small transcendence degree. I," J. Number Theory 25 (1987), no. 3, pp. 279-307.

222. - , "Elliptic curves in two-dimensional abelian varieties and the algebraic independence of certain numbers," Michigan Math. J. 34 (1987), no. 2, pp. 173-182.

223. - , "A Diophantine problem on elliptic curves," Trans. Amer. Math. Soc. 309 (1988), no. 1 , pp. 325-338.

224. - , "Algebraic groups and small transcendence degree. II," J. Number Theory 35 (1990), no. 2, pp. 109-127.

225. K. G. VASIL'EV - "On the algebraic independence of the periods of Abelian integrals," Mat. Zametki 60 (1996), no. 5, pp. 681-691, 799.

226. I. WAKABAYASHI - "Algebraic values of meromorphic functions on Riemann surfaces," J. Number Theory 25 (1987), no. 2, pp. 220-229.

227. - , "Algebraic values of functions on the unit disk," in Prospects of mathematical science (Tokyo, 1986), World Sci. Publishing, Singapore, 1988, pp. 235-266.

228. - , "An extension of the Schneider-Lang theorem," in Seminar on Diophantine Approximation (Japanese) (Yokohama, 1987), Sem. Math. Sci., vol. 12, Keio Univ., Yokohama, 1988, pp. 79-83.

229. M. WALDSCHMIDT - "Propriétés arithmétiques des valeurs de fonctions méromorphes algébriquement indépendantes," Acta Arith. 23 (1973), pp. 19-88. 
230. - , Nombres transcendants, Springer-Verlag, Berlin, 1974, Lecture Notes in Mathematics, Vol. 402.

231. - , "Les travaux de G. V. Chudnovsky sur les nombres transcendants," in Séminaire Bourbaki, Vol. 1975/76, 28e année, Exp. No. 488, Springer, Berlin, 1977, pp. 274-292. Lecture Notes in Math., Vol. 567.

232. - , "Nombres transcendants et fonctions sigma de Weierstrass," C. R. Math. Rep. Acad. Sci. Canada 1 (1978/79), no. 2, pp. 111-114.

233. - , Nombres transcendants et groupes algébriques, Astérisque, vol. 69, Société Mathématique de France, Paris, 1979, With appendices by Daniel Bertrand and JeanPierre Serre.

234. - , "Diophantine properties of the periods of the Fermat curve," in Number theory related to Fermat's last theorem, Proc. Conf., Prog. Math. 26, 79-88, 1982.

235. - , "Algebraic independence of transcendental numbers. Gel'fond's method and its developments," in Perspectives in mathematics, Birkhäuser, Basel, 1984, pp. 551-571.

236. - , "Algebraic independence of values of exponential and elliptic functions," J. Indian Math. Soc. (N.S.) 48 (1984), no. 1-4, pp. 215-228 (1986).

237. - , "Groupes algébriques et grands degrés de transcendance," Acta Math. 156 (1986), no. 3-4, pp. 253-302, With an appendix by J. Fresnel.

238. - , "Some transcendental aspects of Ramanujan's work," in Proceedings of the Ramanujan Centennial International Conference (Annamalainagar, 1987), RMS Publ., vol. 1, Ramanujan Math. Soc., 1988, pp. 67-76.

239. - - "Sur la nature arithmétique des valeurs de fonctions modulaires," Astérisque $\mathbf{2 4 5}$ (1997), p. Exp. No. 824, 3, 105-140, Séminaire Bourbaki, Vol. 1996/97.

240. - , "Density measure of rational points on abelian varieties," Nagoya Math. J. 155 (1999), pp. 27-53.

241. - , "Transcendance et indépendance algébrique de valeurs de fonctions modulaires," in Number theory (Ottawa, ON, 1996), CRM Proc. Lecture Notes, vol. 19, Amer. Math. Soc., Providence, RI, 1999, pp. 353-375.

242. - , "Algebraic independence of transcendental numbers: a survey," in Number theory, Trends Math., Birkhäuser and Hindustan Book Agency, Basel and New-Delhi, 2000, pp. 497-527.

243. - , "Transcendence of periods: the state of the art," Pure and Applied Mathematics Quarterly 2 (2006), no. 2, pp. 199-227.

244. E. WhitTAKer \& G. WATSON - A course of modern analysis. An introduction to the general theory on infinite processes and of analytic functions; with an account of the principal transcendental functions. 4th ed., reprinted, Cambridge: At the University Press. 608 p., 1962.

245. J. WOLFART \& G. WÜSTHOLZ - "Der Überlagerungsradius gewisser algebraischer Kurven und die Werte der Betafunktion an rationalen Stellen,” Math. Ann. 273 (1985), no. 1 , pp. 1-15.

246. G. WÜSTHOLZ - "Algebraische Unabhängigkeit von Werten von Funktionen, die gewissen Differentialgleichungen genügen,” J. reine angew. Math. 317 (1980), pp. 102-119.

247. — , "Sur l'analogue abélien du théorème de Lindemann," C. R. Acad. Sci. Paris Sér. I Math. 295 (1982), no. 2, pp. 35-37.

248. - , "Über das Abelsche Analogon des Lindemannschen Satzes. I," Invent. Math. 72 (1983), no. 3, pp. 363-388.

249. - , "Recent progress in transcendence theory," in Number theory, Noordwijkerhout 1983, Lecture Notes in Math., vol. 1068, Springer, Berlin, 1984, pp. 280-296. 
250. - , "Transzendenzeigenschaften von Perioden elliptischer Integrale," J. reine angew. Math. 354 (1984), pp. 164-174.

251. - , "Algebraische Punkte auf analytischen Untergruppen algebraischer Gruppen," Ann. of Math. (2) 129 (1989), no. 3, pp. 501-517.

252. A. Y. YANCHENKO - "On the measure of algebraic independence of values of derivatives of a modular function (the p-adic case)," Mat. Zametki 61 (1997), no. 3, pp. 431-440, Engl. Transl. Math. Notes 61, 3 (1997), 352-359.

253. K. R. YU - "Linear forms in elliptic logarithms," J. Number Theory 20 (1985), no. 1, pp. 1-69.

254. D. ZAGIER - "Introduction to modular forms," in From Number Theory to Physics (Les Houches, 1989), Springer, Berlin, 1992, pp. 238-291. 